\title{
The Extraordinary Passage of Comet C/2020 F3 NEOWISE: Evidence for Heterogeneous Chemical Inventory in Its Nucleus
}

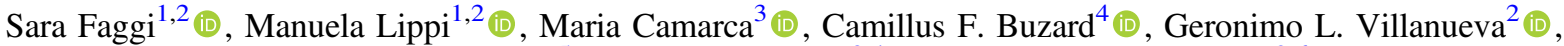 \\ Gregory W. Doppmann ${ }^{5}$, Geoffrey A. Blake ${ }^{3,4}$ (D), and Michael J. Mumma ${ }^{2,6}$ (D) \\ ${ }^{1}$ American University, 4400 Massachusetts Avenue NW, Washington, DC 20016, USA; faggi@american.edu \\ ${ }^{2}$ NASA Goddard Space Flight Center, 8800 Greenbelt Road, MD 20771, USA \\ ${ }^{3}$ Caltech, Division of Geological and Planetary Science, Pasadena, CA 91125, USA \\ ${ }^{4}$ Caltech, Division of Chemistry and Chemical Engineering, Pasadena, CA 91125, USA \\ ${ }^{5}$ W.M. Keck Observatory, HI, USA \\ ${ }^{6}$ University of Maryland, 1940 Regents Drive, College Park, MD 20740, USA \\ Received 2021 March 22; revised 2021 July 22; accepted 2021 July 24; published 2021 October 5
}

\begin{abstract}
Upon its discovery in 2020 March, we requested Director Discretionary Time (DDT) at the NASA/IRTF facility to observe comet C/2020 F3 (NEOWISE), with the high-resolution spectrograph iSHELL. The comet approached the Sun, down to $0.3 \mathrm{au}$, in early 2020 July, providing a spectacular perihelion passage and revealing itself as one of the brightest comets that have appeared in the northern hemisphere in recent decades. Daytime observations with iSHELL/IRTF were performed to study the comet immediately after its perihelion passage, from 9 July to 1 August. In early August, a DDT at Keck Observatory was requested to continue following the comet with NIRSPEC 2.0. We acquired comprehensive high-resolution spectra of the comet as it progressively retreated from perihelion. We detected many cometary emission lines in the (2.8-5.3) $\mu \mathrm{m}$ range, identifying 12 molecular species: 9 primary volatiles $\left(\mathrm{H}_{2} \mathrm{O}, \mathrm{HCN}, \mathrm{NH}_{3}, \mathrm{CO}, \mathrm{C}_{2} \mathrm{H}_{2}, \mathrm{C}_{2} \mathrm{H}_{6}, \mathrm{CH}_{4}, \mathrm{CH}_{3} \mathrm{OH}\right.$, and $\left.\mathrm{H}_{2} \mathrm{CO}\right)$ and 3 product species $(\mathrm{CN}$, $\mathrm{NH}_{2}, \mathrm{OH}$ ). In this paper, we present the analysis and discussion of the molecular abundances found in this comet, and we compare them to reference median values for Oort Cloud Comets. Measured molecular mixing ratios showed drastic changes during our observing campaign, which spanned nearly one month from 2020 July 9 to August 6. Here, we provide a detailed discussion of the molecular spatial distributions and their role in identifying the presence of extended sources in the coma. Our investigations provided evidence for the heterogeneous chemical composition of the comet's nucleus.
\end{abstract}

Unified Astronomy Thesaurus concepts: Comet volatiles (2162); High resolution spectroscopy (2096); Astrobiology (74)

\section{Introduction}

Compositional investigations of cometary nuclei are essential to understanding the formation and evolution of organic matter within the solar system (Mumma et al. 1993; Irvine et al. 2000; Bockelée-Morvan et al. 2004; Mumma \& Charnley 2011). In the past decades, fundamental research on comets emphasized retrievals of volatile abundances as key to understanding the origin of prebiotic matter in comets and the significance of its delivery to planets after planetary formation and migration.

Although cometary icy nuclei resided in cold reservoirs for their entire lives, their observed chemical composition is not completely pristine. Cometary ices might retain heritage signatures from the prestellar core/ISM phase era, but they also experienced the processing that characterized the different evolutional stages of our protoplanetary disk. According to disk models (Bergin et al. 2007; Cleeves et al. 2014; Bergin \& Cleeves 2018; Eistrup et al. 2019), we indeed expect to observe different signatures of processing on cometary ices according to the different birthplace locations in the disk midplane. Without getting too much into details, which would be beyond the scope of this paper, we could expect that the disk physical properties (such as the gas and dust density distributions, the temperature, the energy radiation fields, etc.), and their different location(s) of influence (e.g., the disk midplane or the warm molecular layer), had strongly affected the resulting chemical composition of the gas and the evolution of the dust population. For example, at low temperatures, the gas begins to "freeze out" onto dust surfaces, and both in the midplane and in the molecular layer, it has been seen that some ices can impact the growth of grains by altering their sticking efficiency and seeding the initial phases of planet formation (Wang et al. 2005).

According to the "Nice" model (Jewitt et al. 2007; Morbidelli \& Rickman 2015), after the disruption of the icy debris disk by giant planet migrations, icy nuclei populated both the Oort cloud (OC) and the Kuiper Belt (KB). Once relocated in their distant reservoirs, cometary nuclei experienced fewer collisional events but still persistent cosmic-ray (CR) bombardment along with cryogenic preservation. Cosmic-ray irradiation mainly affected signatures of the cometary surface layer (to depths of $100 \mathrm{~g} \mathrm{~cm}^{-2}$ or so), for both OC and KB objects (Garrod 2019). Following injection into the inner solar system, the subsequent multiple passages around the Sun also introduced surface erosion and possibly thermal processing. The latter is most severe for shortperiod comets, with Jupiter Family and Encke-type Comets being at greatest risk of such processing; compared with longperiod comets, JFCs indeed often show a lack of hypervolatile species as does 2P/Encke (Mumma \& Charnley 2011).

One of the main challenges and fundamental inquiries of cometary science and astrobiology is understanding how well such signatures have been preserved in the cometary ices we study today. Measuring the compositional diversity of cometary nuclei presents a very convoluted problem, as does disentangling the processing histories that such bodies have experienced since formation. However, signatures of interstellar chemistry and 

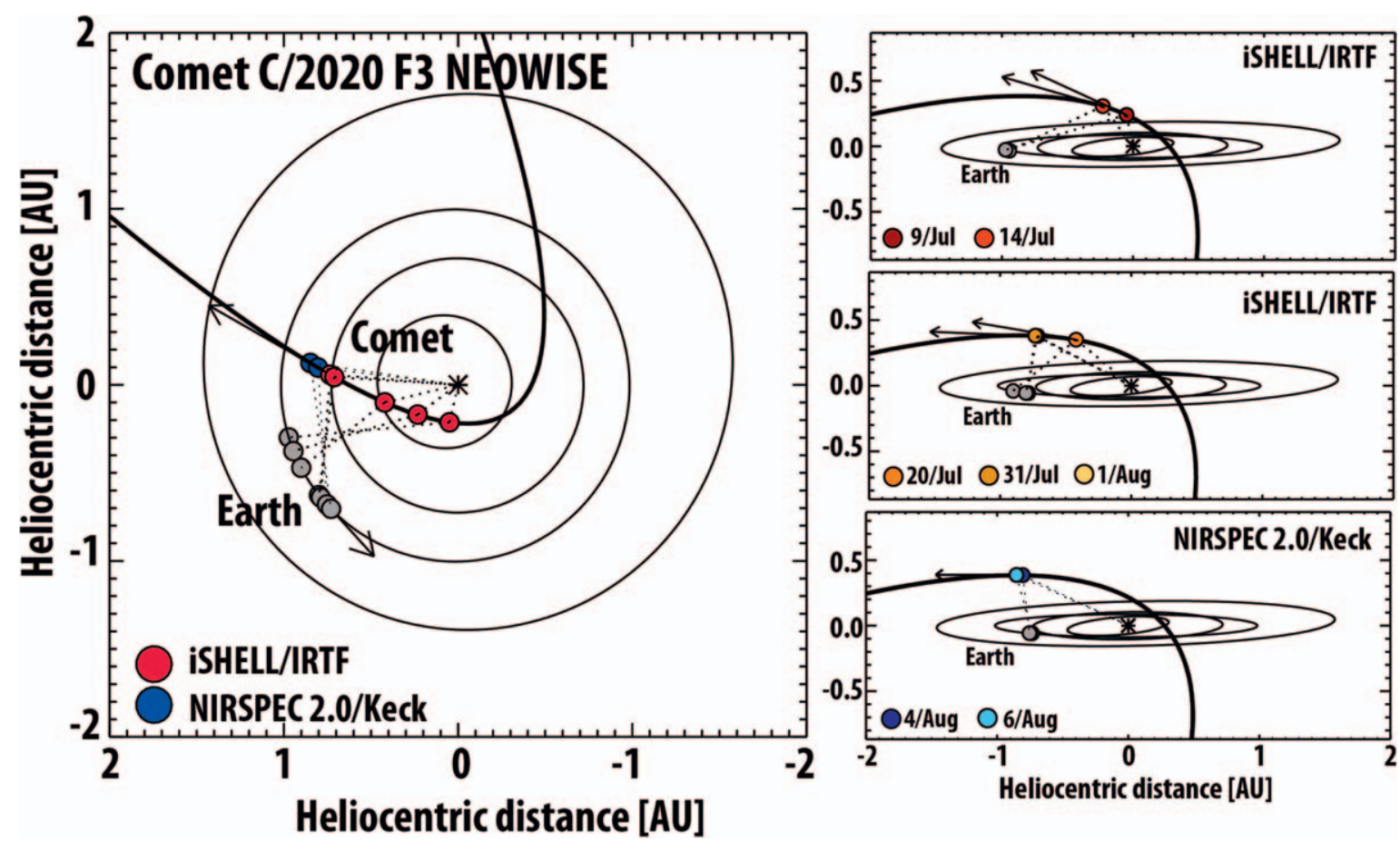

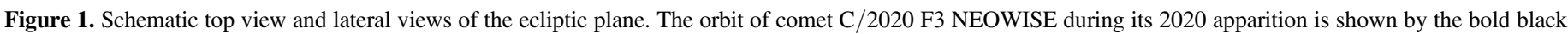

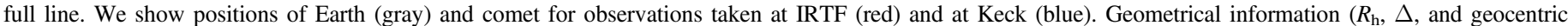
velocity) for selected dates are reported in Table 1.

processing may be preserved in cometary material in the form of cosmogonic indicators (or thermometers). Thus, investigation of such thermometers, both within individual comets and over the cometary population, can provide reliable insights into conditions of the formation and evolution of our planetary system (Mumma \& Charnley 2011; Lippi et al. 2020, 2021).

In this paper, we present investigations of the molecular composition of the long-period comet C/2020 F3 (NEOWISE) conducted with iSHELL at the NASA/IRTF Facility and NIRSPEC $2.0^{7}$ at Keck Observatory. Comet C/2020 F3 (NEOWISE), hereafter comet NEOWISE, was discovered on 2020 March 27 by the WISE space telescope, under the NEOWISE program. It occupies a retrograde near-parabolic orbit that, before entering the inner solar system, had a semimajor axis of $274.5 \mathrm{au}$ with an orbital period of $4548 \mathrm{yr}$ $(1 / \mathrm{a}=0.003642$, Nakano Note 4202). With a modest orbital period and small perihelion distance, NEOWISE likely experienced previous apparitions in the inner solar system. Comet NEOWISE is the brightest comet to appear in the northern hemisphere since the passage of comet Hale-Bopp in 1997, and for this reason, it attracted the attention of both professional and amateur astronomers who widely recorded its approach. The comet emerged from perihelion on 2020 July 3 (at a heliocentric distance $\left(R_{\mathrm{h}}\right)$ of 0.294 au and a geocentric distance $(\Delta)$ of about $1.152 \mathrm{au}$ ) with a visual magnitude of about $\sim 0.5-1 \mathrm{mag}$, making it bright enough to be visible to the naked eye. The comet's closest approach to Earth was on 2020 July $23\left(\Delta=0.692\right.$ and $\left.R_{\mathrm{h}}=0.630 \mathrm{au}\right)$. The favorable proximity of comet NEOWISE to both the Sun and Earth, combined with the unique capabilities of the NASA/IRTF Facility and the Keck Observatory, allowed a comprehensive investigation of its organic composition and evolution.

\footnotetext{
7 For clarity, across the paper we will refer to the recently detector-upgraded NIRSPEC/Keck II as NIRSPEC 2.0 .
}

\section{Observations}

We first observed comet NEOWISE using the long-slit nearinfrared high-resolution immersion echelle spectrograph iSHELL at the NASA/IRTF. iSHELL covers the wavelength range of $1.1-5.3 \mu \mathrm{m}$ with a resolving power of about $\lambda / \Delta \lambda=70,000$ when adopting the narrowest slit width of 0 "! 375 ( 3 pixels wide, considering the 0 ". 125 pixel $^{-1}$ plate scale; Rayner et al. 2012). After its perihelion passage, we observed comet NEOWISE from 2020 July 9 to August 1, in five observing runs (see Figure 1 for the orbital diagram and Table 1 for the observing $\log )$. We followed the comet outbound from a heliocentric distance of $R_{\mathrm{h}}=0.338-0.829$ au and geocentric distance from $\Delta=0.963-0.796$ au. During this interval, the comet was a daytime object with a solar elongation angle that varied from $20^{\circ}$ to $52^{\circ}$. The IRTF uniquely permits daytime investigations and iSHELL offers superior image guider performance, allowing the selection of different image filters-essential to properly follow a comet during daytime.

The data were obtained using the standard ABBA telescope nodding technique and employing two of our usual customized iSHELL spectral settings (L1c, Lp1c), as we discussed in our previous works (Faggi et al. 2018, 2019), and two standard settings (Lp2 and M1). We targeted the nitrile region, focusing especially on detections of water, $\mathrm{HCN}, \mathrm{C}_{2} \mathrm{H}_{2}, \mathrm{NH}_{3}$, and $\mathrm{NH}_{2}$ (2.9-3.0 $\mu \mathrm{m})$ in L1c; the carbon-stretch region $(3.3-3.5 \mu \mathrm{m})$ in Lp1c detecting $\mathrm{CH}_{4}, \mathrm{C}_{2} \mathrm{H}_{6}, \mathrm{H}_{2} \mathrm{CO}$, and $\mathrm{CH}_{3} \mathrm{OH}$; and finally targeting $\mathrm{CO}, \mathrm{CN}$, and $\mathrm{H}_{2} \mathrm{O}$ in $\mathrm{M} 1$. We also dedicated specific attention to investigations of HDO both in setting Lp1c and in setting Lp2; a detailed discussion of the water isotopic ratio in this comet will be presented in a separate paper.

A slit width of about 0.75 , which corresponded to a resolving power of about $\lambda / \Delta \lambda=35,000$, was adopted for all the cometary observations, except on July 14 where a nominal slit width of about 0 ". 375 was instead chosen to enhance the 
Table 1

Comet C/2020 F3 NEOWISE Observing Log: IRTF and Keck Observations

\begin{tabular}{|c|c|c|c|c|c|c|c|c|c|c|}
\hline Date & UT (hh:mm) & Object name & $\begin{array}{l}\text { Spectral } \\
\text { Setting }\end{array}$ & $\begin{array}{l}\text { Exposure Time } \\
\text { (s), coadds }\end{array}$ & $\begin{array}{l}\text { Time on } \\
\text { Source } \\
\text { (minute) }\end{array}$ & $\begin{array}{c}\text { Slit } \\
\text { Width }\left({ }^{\prime \prime}\right)\end{array}$ & Airmass & $R_{\mathrm{h}}(\mathrm{au})$ & $\Delta(\mathrm{au})$ & $\dot{\Delta}\left(\mathrm{km} \mathrm{s}^{-1}\right)$ \\
\hline \multicolumn{11}{|c|}{ iSHELL/IRTF } \\
\hline \multirow[t]{4}{*}{9 Jul } & $01: 06-01: 26$ & $\mathrm{C} / 2020 \mathrm{~F} 3$ & L1c & 60,1 & 16 & 0.75 & $1.50-1.62$ & 0.34 & 0.96 & -59.58 \\
\hline & $01: 38-01: 58$ & $\mathrm{C} / 2020 \mathrm{~F} 3$ & Lp1 & 60,1 & 16 & 0.75 & $1.71-1.89$ & 0.34 & 0.96 & -59.58 \\
\hline & $04: 43-04: 54$ & HR4550 & L1c & 60,1 & & 4.0 & $1.13-1.14$ & $\ldots$ & $\ldots$ & $\ldots$ \\
\hline & $04: 50-04: 47$ & HR4550 & Lp1 & 60,1 & & 4.0 & $1.07-1.06$ & $\cdots$ & $\cdots$ & $\ldots$ \\
\hline \multirow[t]{2}{*}{$14 \mathrm{Jul}$} & 01:09-03:28 & $\mathrm{C} / 2020 \mathrm{~F} 3$ & Lp1c & 60,1 & 48 & 0.375 & $1.37-2.54$ & 0.43 & 0.81 & -43.68 \\
\hline & $04: 37-04: 46$ & HR4550 & Lp1c & 60,1 & & 4.0 & $1.15-1.17$ & $\ldots$ & $\ldots$ & $\ldots$ \\
\hline \multirow[t]{6}{*}{$20 \mathrm{Jul}$} & $00: 22-01: 24$ & $\mathrm{C} / 2020 \mathrm{~F} 3$ & L1c & 60,1 & 48 & 0.75 & $1.13-1.19$ & 0.56 & 0.71 & -15.27 \\
\hline & $02: 00-03: 24$ & $\mathrm{C} / 2020 \mathrm{~F} 3$ & Lp1c & 60,1 & 64 & 0.75 & $1.26-1.56$ & 0.56 & 0.71 & -15.27 \\
\hline & $03: 42-03: 46$ & HR4550 & Lp1c & 60,1 & & 4.0 & $1.10-1.11$ & $\ldots$ & $\ldots$ & $\ldots$ \\
\hline & $03: 49-03: 53$ & HR4550 & L1c & 60,1 & & 4.0 & $1.11-1.12$ & $\ldots$ & $\ldots$ & $\ldots$ \\
\hline & 04:02-04:07 & HR4550 & M1 & 30,2 & & 4.0 & $1.13-1.14$ & $\cdots$ & $\cdots$ & $\ldots$ \\
\hline & $04: 19-04: 31$ & $\mathrm{C} / 2020 \mathrm{~F} 3$ & M1 & 3,10 & 20 & 0.75 & $1.94-2.06$ & 0.56 & 0.71 & -15.7 \\
\hline \multirow[t]{4}{*}{$31 \mathrm{Jul}$} & $01: 13-01: 56$ & $\mathrm{C} / 2020 \mathrm{~F} 3$ & L1c & 60,1 & 32 & 0.75 & $1.04-1.02$ & 0.81 & 0.77 & 33.28 \\
\hline & $02: 11-06: 49$ & $\mathrm{C} / 2020 \mathrm{~F} 3$ & Lp2 & 60,1 & 96 & 0.75 & $1.01-2.20$ & 0.81 & 0.77 & 33.28 \\
\hline & $05: 28-05: 30$ & HR4983 & L1c & 30,1 & & 4.0 & $1.22-1.23$ & $\cdots$ & $\cdots$ & $\ldots$ \\
\hline & $05: 38-05: 40$ & HR4983 & Lp2 & 30,1 & & 4.0 & $1.26-1.27$ & $\ldots$ & $\ldots$ & $\ldots$ \\
\hline \multirow[t]{5}{*}{1 Aug } & $00: 42-03: 25$ & $\mathrm{C} / 2020 \mathrm{~F} 3$ & Lp2 & 60,1 & 112 & 0.75 & $1.08-1.05$ & 0.83 & 0.80 & 35.86 \\
\hline & 06:01-06:50 & & & & & & $1.61-2.17$ & & & \\
\hline & $03: 52-04: 02$ & HR4983 & Lp2 & 60,1 & & 4.0 & $1.04-1.05$ & $\ldots$ & $\cdots$ & $\ldots$ \\
\hline & $04: 21-04: 27$ & HR4983 & M1 & 15,2 & & 4.0 & $1.07-1.06$ & $\ldots$ & $\ldots$ & $\ldots$ \\
\hline & $04: 46-05: 39$ & $\mathrm{C} / 2020 \mathrm{~F} 3$ & M1 & 10,6 & 40 & 0.75 & $1.22-1.46$ & 0.83 & 0.80 & 33.86 \\
\hline \multicolumn{11}{|c|}{ NIRSPEC 2.0/Keck II } \\
\hline \multirow[t]{2}{*}{4 Aug } & $5: 44-5: 54$ & $\mathrm{C} / 2020 \mathrm{~F} 3$ & $\mathrm{KL}^{\mathrm{a}}$ & 60,1 & 8 & $0.288^{c}$ & $1.44-1.51$ & 0.90 & 0.87 & 42.99 \\
\hline & $6: 59-7: 01$ & HIP70384 & $\mathrm{KL}^{\mathrm{a}}$ & 30,1 & & $0.288^{\mathrm{c}}$ & $1.44-1.46$ & $\ldots$ & $\ldots$ & $\ldots$ \\
\hline \multirow[t]{2}{*}{6 Aug } & $5: 44-6: 16$ & $\mathrm{C} / 2020 \mathrm{~F} 3$ & $\mathrm{KL}^{\mathrm{b}}$ & 60,1 & 24 & $0.432^{c}$ & $1.42-1.65$ & 0.94 & 0.92 & 46.28 \\
\hline & $6: 23-6: 28$ & HR5072 & $\mathrm{KL}^{\mathrm{b}}$ & 10,1 & & $0.432^{c}$ & $1.60-1.63$ & $\ldots$ & $\ldots$ & $\ldots$ \\
\hline
\end{tabular}

Notes.

${ }^{\mathrm{a}}$ Echelle and cross-disperser positions were set to: $64.2 / 33.2$.

b Echelle and cross-disperser positions were set to: $64.2 / 33.1$.

c We selected a 24 " slit length.

contrast between the cometary emission lines and the bright continuum flux. The slit PA (position angle) was oriented along the extended Sun-comet radius vector direction in all five observing runs (see Figure 2 and Table 2). During our observations, solar phase angles ranged from $93^{\circ}$ to $78^{\circ}$, which, being close to $90^{\circ}$, allowed the heliocentric radius vector (the Sun-to-comet line vector) to lie almost in the plane of the sky.

Observations with Keck were conducted using the recently upgraded long-slit near-infrared high-resolution spectrometer, NIRSPEC 2.0 (McLean et al. 1998; Martin et al. 2018). It covers a wavelength range from 0.95 to $5.5 \mu \mathrm{m}$ with a resolving power of about $\lambda / \Delta \lambda=25,000$ when using the slit width of 0 " 432 (4.5 pixels wide, considering 0 "'096 pixel $^{-1}$ plate scale). Comet NEOWISE was observed on 2020 August 4 and 6 at the beginning of each night, just after dusk (see Figure 1 and Table 1 for the observing parameters).

The standard ABBA on-slit nodding technique was adopted to obtain the data. Despite the modest time on source (see Table 1), we achieved robust measurements for seven parent volatiles, owing to the advantage of the large collecting area offered by the $10 \mathrm{~m}$ telescope. Employing the KL1 setting, we detected water, $\mathrm{C}_{2} \mathrm{H}_{2}, \mathrm{NH}_{3}, \mathrm{CH}_{4}, \mathrm{C}_{2} \mathrm{H}_{6}, \mathrm{H}_{2} \mathrm{CO}$, and $\mathrm{CH}_{3} \mathrm{OH}$. We adopted the nominal slit width of 0 !' 288 (corresponding to a resolving power of about $\lambda / \Delta \lambda=37,500$ ) on the first night and a slit width of 0 ". 432 (corresponding to a resolving power of about $\lambda / \Delta \lambda=25,000)$ on the second night. Again, we positioned the slit (slit PA) along the Sun-comet position angle. For completeness, it is important to note that on August 4 , due to technical problems, the slit rotator motor was set in stationary mode (i.e., not moving), so the field of view on the sky rotated slowly about the slit center as the telescope tracked the comet. Hence, we spanned a small range in position angles for the slit (see Table 2). On August 6 instead, the rotator was set in tracking mode, keeping the slit at a fixed PA on the sky.

During each observing run, infrared flux standard stars were also observed, using the same cometary spectral settings mentioned above. For iSHELL observations, we adopted the wider slit of 4!"0 to minimize the slit losses and properly calibrate the stellar continuum. For NIRSPEC 2.0 observations, the selection of a wider slit width automatically reduces the slit length, so we maintained the same configuration as for the comet to allow proper spectral order cropping and extraction. Correction factors for the total flux were obtained from the spatial intensity distribution measured along the slit. In Table 1 we report a summary of our observations, compiling the spectral settings we used during the different observing runs, the exposure times we adopted, and the comet orbital information. 
Wavelength [um]
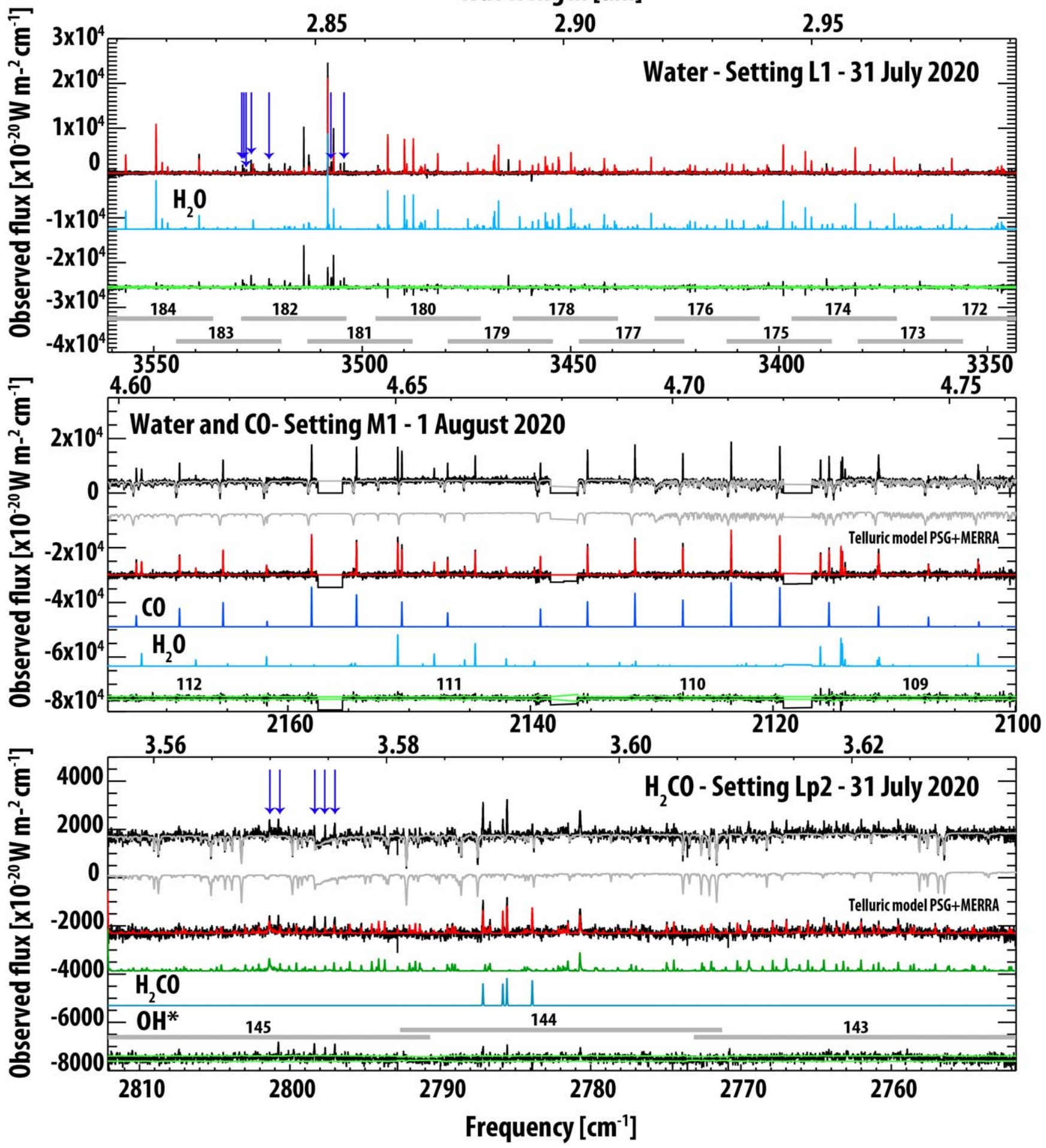

(a)

Figure 2. (a) Spectra extracted from iSHELL/IRTF observations are compared with modeled spectra and residuals: (upper) example of water spectrum extracted from the 12 spectral orders of setting L1c. Unidentified lines are marked with blue arrows. (Middle) CO and water detections from the combination of four spectral orders of setting M1. (Bottom) $\mathrm{H}_{2} \mathrm{CO}$ and $\mathrm{OH}^{*}$ detections from the combination of three spectral orders of setting Lp2. Unidentified lines are marked with blue arrows. (See details in Section 3.1). Telluric is fitted with PSG, adopting the atmospheric and climatological repository MERRA (Gelaro et al. 2017; https://gmao.gsfc.nasa.gov/ reanalysis/MERRA-2/). (b) Zoomed portion of spectra extracted from iSHELL observations: (left) zoomed spectra from order 179 in setting L1 where we detected water. In this example, we show the strong continuum. (Middle-left) Zoomed spectra from order 155 in setting $\mathrm{Lp} 1$ where we detected $\mathrm{C}_{2} \mathrm{H}_{6}, \mathrm{CH}_{4}, \mathrm{weak}_{\mathrm{CH}} \mathrm{OH}$, and $\mathrm{OH}^{*}$. (Middle-right and right) Two zoomed portions of spectra extracted from order 171 in setting L1c where we detected $\mathrm{HCN}_{2} \mathrm{C}_{2} \mathrm{H}_{2}, \mathrm{NH}_{3}, \mathrm{NH}_{2}, \mathrm{H}_{2} \mathrm{O}$, and $\mathrm{OH}^{*}$. Unidentified lines are marked with blue arrows. (c) Spectra extracted from NIRSPEC 2.0/Keck observations: (left) detections of $\mathrm{C}_{2} \mathrm{H}_{6}, \mathrm{CH}_{3} \mathrm{OH}$, and $\mathrm{CH}_{4}$ from order 23 of the KL1 setting. (Right) Detections of water and $\mathrm{NH}_{3}$ from order 26 of the KL1 setting. One unidentified line is marked with a blue arrow. (See details in Section 3.1). 

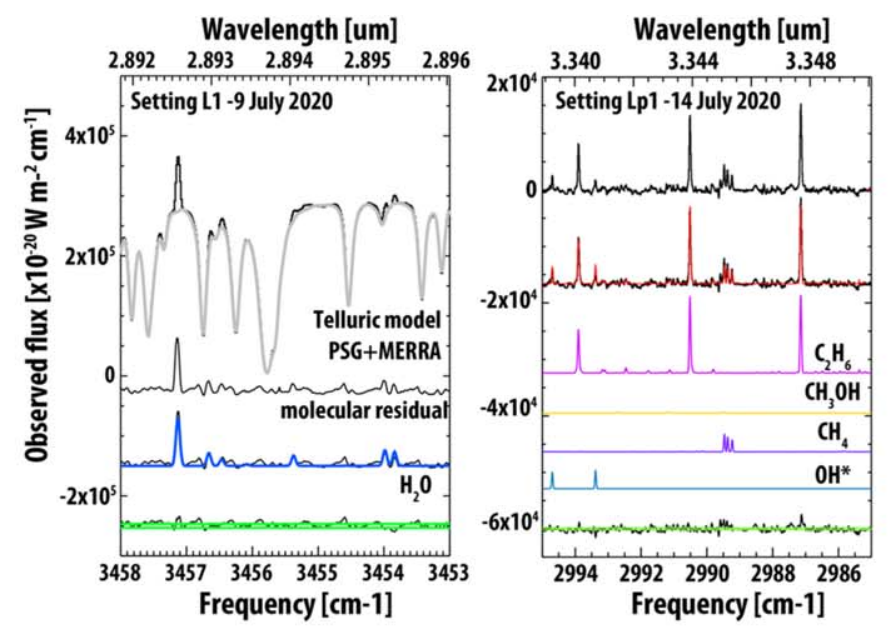
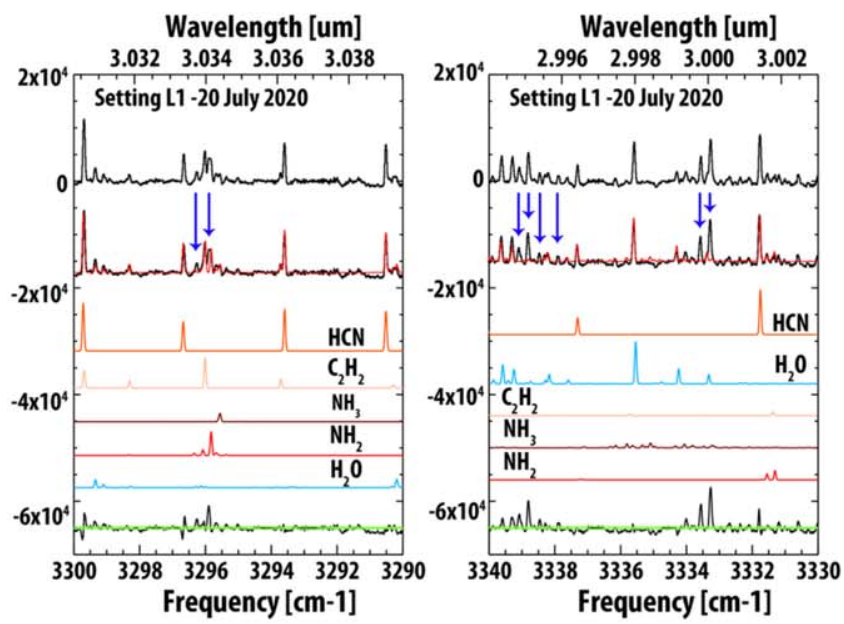

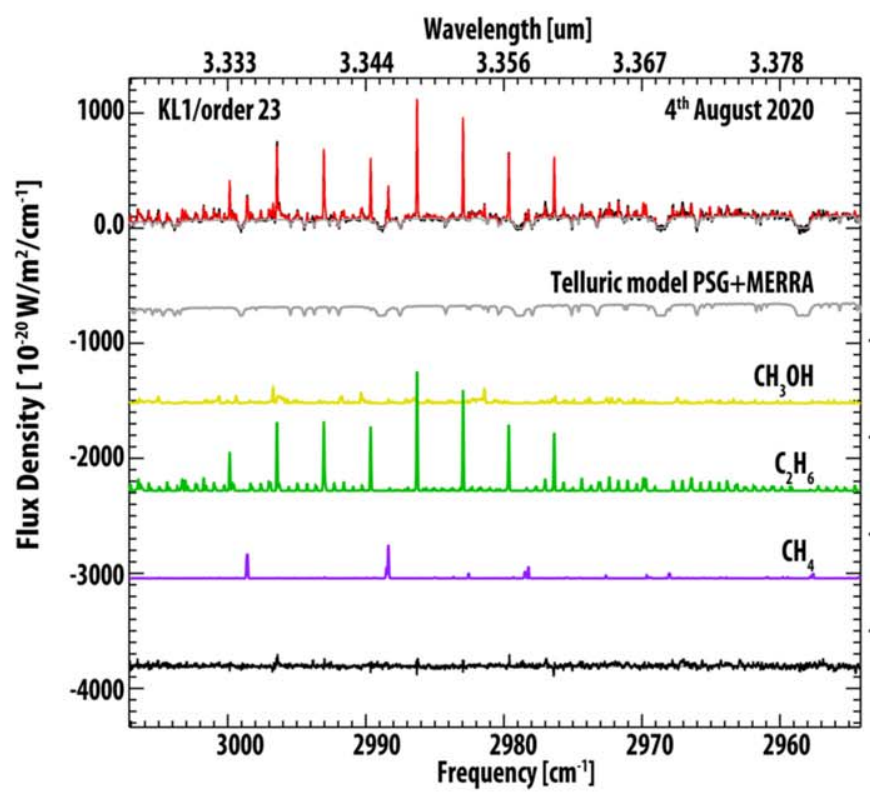

(c)

(b)

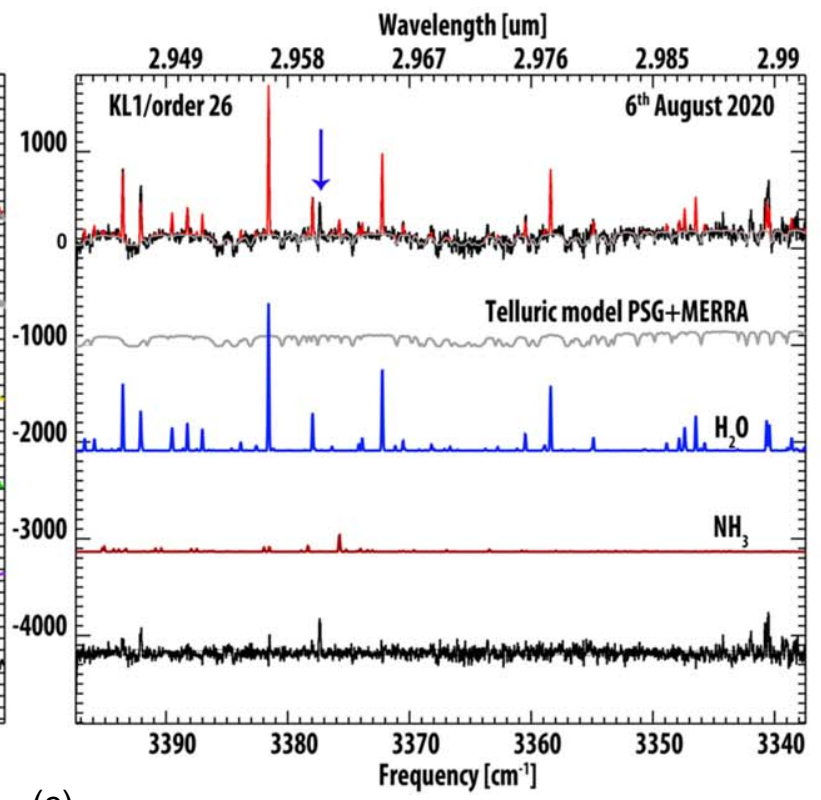

Figure 2. (Continued.)

\section{Results}

\subsection{Extraction of Cometary Spectra}

In the last decade, our team significantly improved dataprocessing algorithms for processing high-resolution cometary data (e.g., DiSanti et al. 2006; Bonev et al. 2009; Villanueva et al. 2012a, 2012b). Thus, using the latest version of our Goddard custom algorithms for data reduction and analysis (see Villanueva et al. 2013), tailored also for iSHELL and NIRSPEC 2.0, we processed the cometary raw frames as follows: each single order of a specific spectral setting was cropped, cleaned, spatially and spectrally straightened, and wavelength- and flux-calibrated. Frequency calibration was obtained by fitting the terrestrial radiance with the Planetary Spectrum Generator (PSG; Villanueva et al. 2018), while the flux calibration was obtained by applying the wavenumberdependent conversion efficiency derived by comparing the top-of-atmosphere stellar flux density $\left(\mathrm{W} \mathrm{m}^{-2} \mathrm{~cm}^{-1}\right)$, modified by the modeled atmospheric transmittance and the measured instrumental counts per pixel (ADU s $\mathrm{s}^{-1}$ ) at individual frequencies across the order. Once processed, we extracted the final calibrated cometary spectra, which we consider as "nucleus-centered" spectra, as follows:

For iSHELL/IRTF data acquired with the 0!"75 slit, we extracted the signal over 15 pixel rows along the slit, corresponding to \pm 7 pixels from the nucleus-centered pixel position. In this case, the signal was contained in a rectangular aperture size $(0$ ". $75 \times 2$ ". 505$)$. For observations on July 14 , we adopted a slit width of about 0 !" 375 , so the cometary spectra were extracted over 9 pixel rows along the slit, corresponding to \pm 4 pixels from the nucleus-centered pixel. In this case, the signal was contained in a rectangular aperture size $(0$ ". $375 \times 1$ ". 494).

For NIRSPEC/Keck data, acquired on August 4 with a slit width of about 0 !' 288 , we extracted the signal over 9 pixel rows along the slit, which correspond to \pm 4 pixels from the nucleuscentered pixel position. In this case the signal was contained in a rectangular aperture of size $(0$ "' $288 \times 1$ !" 170$)$. For the observations on August 6 where we adopted a slit width of about 0 ." 432 , the cometary spectra were extracted over 15 pixel 
Table 2

Spatial Profile Information for Primary Volatiles

\begin{tabular}{|c|c|c|c|c|c|c|c|c|c|}
\hline Date & $\begin{array}{l}\text { Spectral } \\
\text { Setting }\end{array}$ & $\begin{array}{l}\mathrm{Ny}^{\mathrm{a}} \\
\text { (pix) }\end{array}$ & $\Delta$ Gas-Dust $^{\mathrm{b}}$ (pix) & PsAng $^{c}$ (deg) & $\begin{array}{l}\text { PhaseAng }^{\mathrm{d}} \\
\quad(\mathrm{deg})\end{array}$ & $\begin{array}{l}\text { Plate Scale }{ }^{\mathrm{e}}(\operatorname{arc}- \\
\mathrm{sec} / \mathrm{pix})\end{array}$ & $D^{\mathrm{f}}(\operatorname{arcsec})$ & $\Delta(\mathrm{au})$ & $\rho^{\mathrm{g}}(\mathrm{km})$ \\
\hline \multicolumn{10}{|c|}{ iSHELL/IRTF } \\
\hline \multirow[t]{2}{*}{$9 \mathrm{Jul}$} & L1c & 15 & $\mathrm{H}_{2} \mathrm{O}: 4 \mathrm{HCN}: 4$ & 325 & 92.5 & 0.167 & 2.505 & 0.963 & \pm 877.1 \\
\hline & Lp1 & 15 & $\mathrm{C}_{2} \mathrm{H}_{6}: 4$ & & & 0.166 & 2.490 & & \pm 871.9 \\
\hline $14 \mathrm{Jul}$ & Lp1c & 9 & $\mathrm{C}_{2} \mathrm{H}_{6}: 9 \mathrm{CH}_{4}: 9$ & 355 & 107 & 0.166 & 1.494 & 0.809 & \pm 439.5 \\
\hline \multirow[t]{3}{*}{$20 \mathrm{Jul}$} & L1 & 15 & $\begin{array}{c}\mathrm{H}_{2} \mathrm{O}: 13 \mathrm{HCN}: 6 \mathrm{C}_{2} \mathrm{H}_{2}: 6 \\
\mathrm{NH}_{2}: 6,0\end{array}$ & 40.5 & 106 & 0.167 & 2.505 & 0.705 & \pm 642.1 \\
\hline & Lp1c & 15 & $\begin{array}{c}\mathrm{C}_{2} \mathrm{H}_{6}: 4 \mathrm{CH}_{4}: 4 \mathrm{CH}_{3} \mathrm{OH}: 4 \\
\mathrm{H}_{2} \mathrm{CO}: 4\end{array}$ & & & 0.166 & 2.490 & & \pm 638.3 \\
\hline & M1 & 15 & $\mathrm{CO}: 4 \mathrm{H}_{2} \mathrm{O}: 4$ & & & 0.165 & 2.475 & & \pm 634.5 \\
\hline \multirow[t]{2}{*}{$31 \mathrm{Jul}$} & L1 & 15 & $\begin{array}{c}\mathrm{H}_{2} \mathrm{O}: 0 \mathrm{HCN}: 0 \mathrm{C}_{2} \mathrm{H}_{2}: 0 \\
\mathrm{NH}_{2}: 0, \text { asym }\end{array}$ & 90.5 & 80 & 0.167 & 2.505 & 0.796 & \pm 705.0 \\
\hline & Lp2 & 15 & $\mathrm{H}_{2} \mathrm{CO}: 0$ & & & 0.174 & 2.609 & & \pm 734.5 \\
\hline \multirow[t]{3}{*}{1 Aug } & M1 & 15 & CO: 0 asym $\mathrm{H}_{2} \mathrm{O}: 0$ asym & 92.5 & 78 & 0.165 & 2.475 & 0.796 & \pm 716.4 \\
\hline & Lp2 & 15 & $\mathrm{H}_{2} \mathrm{CO}: 0$ & & & 0.174 & 2.609 & & \pm 755.4 \\
\hline & \multicolumn{9}{|c|}{ NIRSPEC/Keck II } \\
\hline 4 Aug & KL1 & 9 & $\begin{array}{c}\mathrm{H}_{2} \mathrm{O}: 0 \mathrm{C}_{2} \mathrm{H}_{6}: 0 \mathrm{CH}_{4}: 0 \\
\mathrm{CH}_{3} \mathrm{OH}: 0\end{array}$ & $96.13-93.83$ & 71 & 0.130 & 1.17 & 0.866 & \pm 368.4 \\
\hline 6 Aug & KL1 & 15 & $\begin{array}{c}\mathrm{H}_{2} \mathrm{O}: 0 \mathrm{C}_{2} \mathrm{H}_{6}: 0 \mathrm{CH}_{4}: 0 \\
\mathrm{CH}_{3} \mathrm{OH}: 0\end{array}$ & 98.05 & 67 & 0.130 & 1.950 & 0.918 & \pm 650.9 \\
\hline
\end{tabular}

Notes.

${ }^{a}$ Number of pixels along the slit, centered on the gas peak position, from which we extracted the nucleus-centric cometary spectra.

b Pixels shift between the gas and the dust peaks along the slit.

${ }^{c}$ Slit position angle, which was set to be the same as the Sun position angle; see Figure 2.

d Solar phase angles.

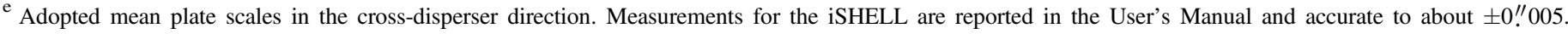
Measurements for NIRSPEC 2.0 are reported at https://www2.keck.hawaii.edu/inst/nirspec/.

f Spanned arcsecond along the slit.

${ }^{\mathrm{g}}$ Corresponding projected nucleocentric distance in kilometer sampled along the slit.

rows along the slit, corresponding to \pm 7 pixels from the nucleus-centered position. In this case the signal was contained in a rectangular aperture of size of $(0$ " $375 \times 1$ "! 950$)$.

Details on the spectral extractions for both iSHELL and NIRSPEC 2.0 data are reported in Table 2, along with information on spatial profiles. Examples of cometary spectra are shown in Figures 2(a), (b), and (c), and compared with optimized synthetic spectra for detected species. During the first three dates (July 9, 14, and 20) the cometary continuum flux density was very strong and the contrast with the molecular emission lines was poor. For this reason, on those dates we present the molecular residual spectra after the continuum subtraction. From 2020 July 31, the cometary continuum was less bright compared to earlier dates and molecular emission lines show improved contrast, so we decided to present the extracted spectra before cometary continuum subtraction-examples are reported in the middle panel of Figure 2(a) (iSHELL) and in Figure 2(c) (NIRSPEC). The cometary continuum, affected by terrestrial atmospheric transmittance, was synthesized with PSG (Villanueva et al. 2018). In Figure 2(a), it is shown (in gray) superimposed and shifted below the measured spectrum. Examples of cometary molecular residuals (after continuum subtraction) and of the modeled fluorescent emission spectra for detected molecules $\left(\mathrm{H}_{2} \mathrm{O}, \mathrm{HCN}, \mathrm{C}_{2} \mathrm{H}_{2}, \mathrm{NH}_{3}, \mathrm{NH}_{2}, \mathrm{C}_{2} \mathrm{H}_{6}, \mathrm{CH}_{4}, \mathrm{H}_{2} \mathrm{CO}\right.$, and $\left.\mathrm{CO}\right)$ are reported in the middle traces of the graphics (using different colors). The lowest trace shows the spectral residuals after subtracting the modeled molecular detections; the $1 \sigma$ error level is displayed in bright green.
The topmost graphic in Figure 2(a) reports spectra extracted on 2020 July 31 . We show the combination of 12 orders across the setting L1c of iSHELL sampling water from order 184 to order 172; 133 water lines were detected on that day, and some weak unidentified lines (marked with blue arrows) are most likely associated with $\mathrm{OH}^{*}$ prompt emission lines which were not present in the HITRAN line list adopted to identify $\mathrm{OH}$ lines. In the middle graphic, we show the spectra extracted on 2020 August 1 where 18 CO rovibrational emission lines were identified within four spectral orders of the M1 setting of iSHELL, from order 112 to order 109; multiple water lines are also present. The bottom graphic of Figure 2(a) reports detection of formaldehyde and $\mathrm{OH}^{*}$ (prompt emission) within three spectral orders of setting Lp2 of iSHELL, orders 142, 144 , and 145 , on 2020 July 31 . Unidentified lines are marked and they might be $\mathrm{OH}^{*}$ lines not present in the line list.

In Figure 2(b), we show four zoomed sections. The leftmost panel reports part of order 179 in the L1 setting on 2020 July 9. The middle-left graphic shows an extract of order 155 in the Lp1 setting on 2020 July 14, while the middle-right and rightmost panels show two sections of order 171 in the L1 setting on 2020 July 20 . In the leftmost panel (order 179/L1) we show the strong continuum detected in comet NEOWISE along with the detection of water lines. In the middle-left panel (order 155/Lp1), strong detections of ethane, methane, and $\mathrm{OH}^{*}$ are present; methanol appears barely visible, while in the last two panels on the right (order 171/L1) strong detections of $\mathrm{HCN}, \mathrm{H}_{2} \mathrm{O}, \mathrm{C}_{2} \mathrm{H}_{2}$, and $\mathrm{NH}_{2}$ are present along with one weak line of $\mathrm{NH}_{3}$. Many unidentified lines appear also in this spectral region and are marked with blue arrows. We hypothesize again 
that unidentified lines might be $\mathrm{OH}^{*}$ prompt emission lines that were not present in the adopted line list, as already mentioned above, or alternatively, we could think about prompt emission or hot-band lines from $\mathrm{NH}_{2}$ or ammonia. Further discussion is deferred to a future paper.

In Figure 2(c), spectra extracted on 2020 August 4 and 6, from setting KL1 of NIRSPEC 2.0, are shown. Very strong detections of $\mathrm{CH}_{3} \mathrm{OH}, \mathrm{C}_{2} \mathrm{H}_{6}$, and $\mathrm{CH}_{4}$ extracted from order 23 are reported in the left panel, while water and ammonia from order 26 are shown in the right panel. One unidentified line is marked.

\subsection{Molecular Spatial Profiles}

During the spectral extraction process and the associated spatial profile analysis, we identified interesting features of the molecular outflow and the dust profile especially on dates closer to the perihelion passage. Spatial profile distributions along the slit, extracted from the brightest emission lines, are reported in Figure 3(a) and detailed parameters are reported in Table 2. In Figure 3(a), each row is associated with a specific observing run. The adopted slit position angles are reported in the first column (also reported in the fifth column of Table 2). We set the slit PA along the Sun-comet position angle directions to investigate the release of material (either gas and dust) in the sunward and antisunward directions.

Spatial profiles for individual molecular species and for dust are reported in the main grid of Figures 3(a) and (b). Each molecule is displayed with a distinct color, which is kept the same throughout the manuscript to assist visualization. Progressing from the second column to the last in the main grid of Figure 3(a), we report $\mathrm{H}_{2} \mathrm{O}$ in light blue, $\mathrm{HCN}$ in orange, $\mathrm{NH}_{2}$ in red, $\mathrm{C}_{2} \mathrm{H}_{2}$ in light pink, $\mathrm{C}_{2} \mathrm{H}_{6}$ in light purple, $\mathrm{CH}_{4}$ in dark purple, $\mathrm{CH}_{3} \mathrm{OH}$ in yellow, $\mathrm{H}_{2} \mathrm{CO}$ in green, and $\mathrm{CO}$ in dark blue. We also label each spectrum to assist the color-challenged reader.

Shaded gray areas identify the regions outside the combination of the $\mathrm{A}$ and the $\mathrm{B}$ beam. Beyond the combined center region, profiles are discarded because they are more susceptible to systematics.

Looking at the spatial profiles we can identify two main behaviors: in the first three observing days (July 9, 14, and 20), all molecular profiles are shifted toward the antisunward direction, relative to the dust profiles that are centered on the (targeted) nucleocentric position. On the latter four observing dates (July 31 and August 1, 4, and 6) the spatial profiles for all gas primary species follow the dust distribution. Importantly, as mentioned above we targeted solar phase angles close to $90^{\circ}$ allowing the Sun-comet vector to be nearly in the plane of the sky and therefore minimizing projection effects on the observed spatial profiles.

Usually, enhancements of the molecular spatial profiles in diverse specific directions have been interpreted as evidence for gaseous release directly from the nucleus or release from either icy grains or small refractory grains carried into the coma. For example, in comets such as C/2013 V5 (Oukaimeden; DiSanti et al. 2018), C/2007 W1 Boattini (Villanueva et al. 2011b), C/2009 P1 Garradd (Villanueva et al. 2012c), and 103P/Hartley 2 (A'Hearn et al. 2011; Mumma et al. 2011), this scenario has been widely discussed and characterized (comparison with comet NEOWISE is reported in the Discussion section).

Focusing on each observing run we notice the following behaviors: on July 9, the gas-to-dust shifts (Table 2) are the same for the detected gases $\left(\mathrm{H}_{2} \mathrm{O}, \mathrm{HCN}, \mathrm{NH}_{2}\right.$, and $\left.\mathrm{C}_{2} \mathrm{H}_{6}\right)$ and the measured relative pixel shift values are reported in Table 2; on July 14 due to bad weather conditions, we were able only to observe the comet in the Lp1c setting, missing a direct water measurement for that date; however, $\mathrm{OH}^{*}$ (prompt emission) tracks the spatial distribution for $\mathrm{H}_{2} \mathrm{O}$ (its precursor; Bonev et al. 2004), so we show the $\mathrm{OH}^{*}$ spatial profile for a line near $2994 \mathrm{~cm}^{-1}$. The gas-to-dust shifts are the same for the three molecules $\left(\mathrm{OH}^{*}, \mathrm{CH}_{4}\right.$, and $\left.\mathrm{C}_{2} \mathrm{H}_{6}\right)$ detected on July 14 , but they differ from the offsets seen on July 9.

Observations conducted on July 20 sampled three spectral settings (L1, Lp1, and M1) across the 2.9-5 $\mu \mathrm{m}$ wavelengths region, providing a more complete picture of comet NEOWISE composition and spatial analysis. We investigated spatial profiles for all detected species, except for ammonia whose fluorescent emission lines were too weak to enable reliable spatial analysis. We therefore showed the profiles of $\mathrm{NH}_{2}$ that is usually thought to be produced by the photodissociation of ammonia in the cometary coma (Kawakita \& Mumma 2011; Shinnaka \& Kawakita 2016) and whose spatial profiles might qualitatively inform on ammonia, although a photodissociation coma model would be necessary to confirm it quantitatively. Focusing on July 20, we see that the molecular profile shifts appear (somewhat) different for individual species (see Figure 3(b), lower panels). Starting with setting L1c/iSHELL $(2.9-3.1 \mu \mathrm{m})$, we observed that the gas-to-dust shift of $\mathrm{H}_{2} \mathrm{O}$ differs from that of $\mathrm{HCN}, \mathrm{C}_{2} \mathrm{H}_{2}$, and $\mathrm{NH}_{2}$. The water profile is more shifted than that of the other molecules, with a shift value of 13 pixels in the antisunward direction relative to the dust peak. Both $\mathrm{HCN}$ and $\mathrm{C}_{2} \mathrm{H}_{2}$ are shifted by 6 pixels (Figure 3(b), first left lower panel) while $\mathrm{NH}_{2}$ shows an interesting, peculiar case in which we observed the possible presence of more than one component characterizing its spatial distribution: one that is consistent with the gas shifted $\sim 6$ pixels in the antisunward direction (in agreement with $\mathrm{HCN}$ and $\mathrm{C}_{2} \mathrm{H}_{2}$ spatial profiles), producing its broader profile in that direction, and another that is consistent with the dust profile (Figure 3(b), second lower panel).

The origin of the first $\mathrm{NH}_{2}$ component might be associated with a parent volatile in the gas coma, for example, radical $\mathrm{NH}_{2}$ might be created as a photodissociation product of ammonia in the gas phase. However, if $\mathrm{NH}_{2}$ was produced by the sole dissociation of $\mathrm{NH}_{3}$ we would expect a profile that certainly was broader than the parent profile but probably with its peak shifted from the dust peak as seen in the other species. Interestingly, the $\mathrm{NH}_{2}$ profile also overlaps the dust profile in the sunward direction, pointing to a qualitatively possible additional source.

The principal mechanism for excitation of the amidogen radical in the coma is by solar fluorescence. However, as discussed in Kawakita \& Mumma (2011) for comet Machhloz, it cannot be completely ruled out that radical $\mathrm{NH}_{2}$ might be also vibrationally and/or rotationally excited in prompt emission, as in the case of $\mathrm{OH}^{*}$, after its formation in the coma by photodissociation of a parent molecule, whether such parent is ammonia (95\% efficiency) or another species (e.g., $\mathrm{N}_{2} \mathrm{H}_{4}, \mathrm{CH}_{3} \mathrm{NH}_{2}$.). In this case, the spatial profile of the amidogen radical would be proportional to the column density of such parent, and its prompt emission could be used as a proxy for the parent abundance. We do not know whether prompt emission from highly excited rovibrational states of $\mathrm{NH}_{2}$ is present in comet NEOWISE, but what we observed is that its spatial profiles follow the dust profiles in the sunward direction, and this qualitatively indicates a possible additional source related to the dust.

The origin of this second component might be connected to solid-phase material, for example, $\mathrm{NH}_{2}$ might be released as a 
9/Jul

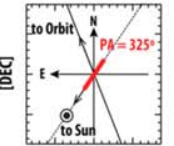

$14 / J u l$

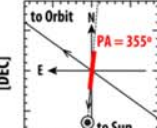

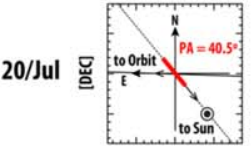

$31 / \mathrm{Jul}$

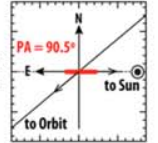

1/Aug

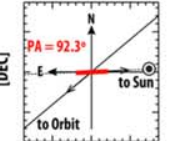

4/Aug

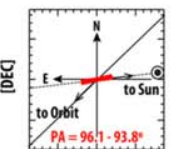

6/Aug

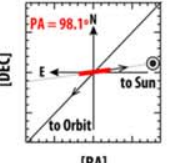

[RA]
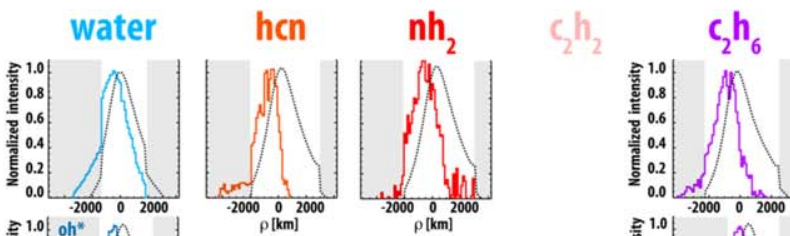

$\mathbf{c h}_{4} \quad \mathrm{Ch}_{3} \mathrm{oh} \quad \mathbf{h}_{2} \mathbf{c o}$

CO
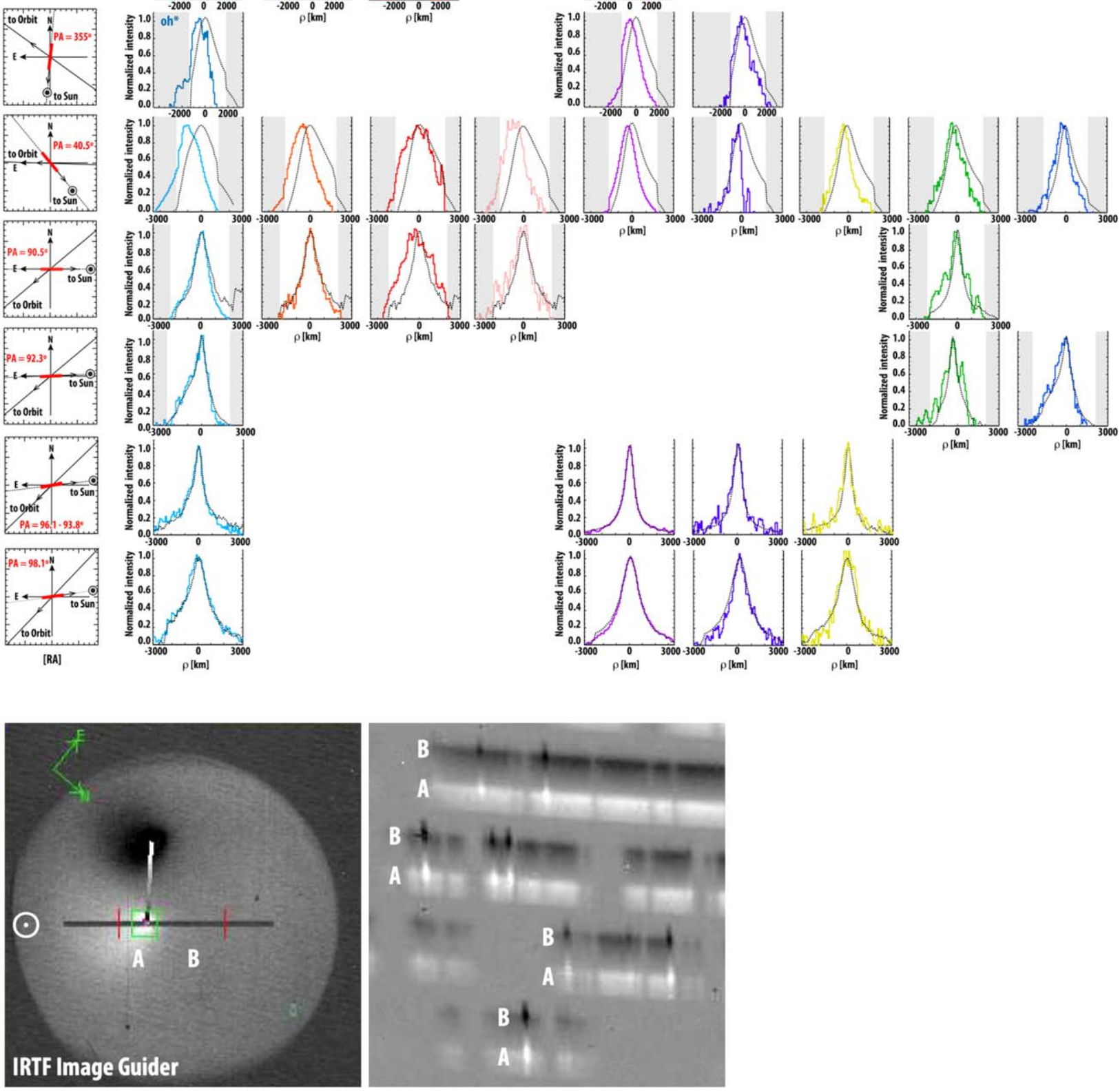

(a)

Figure 3. (a) Upper plot. The leftmost graphics show the adopted slit position angles during each observing run. Slit PAs were set along the Sun-to-comet radial direction. The main grid shows the molecular spatial profiles (various colors) and dust spatial profiles (black dotted) during the observing campaign. Positive $\rho$ (km) are in the Sun direction. Shaded gray areas mark the regions outside the combination of the A and the B beams. Beyond this "good" center region, where a proper A-B sky cancellation is obtained, the profiles are more susceptible to systematics and are discarded. Lower images. Left: example of coma image (dust, $3.5 \mu \mathrm{m})$ taken by IRTF image guider during observations of July 20. Middle: zoomed 2D echellogram extract showing water emission lines in the L1c setting. The gas-to-dust profile shifts, and asymmetries are notable in the 2D raw frame. (b) Upper panels: comparison of dust profiles at various wavelengths on 2020 July 20 . Stellar profiles are also shown in black lines for comparison. Dust profiles are here shown as dotted colored lines, and each color is linked to the associated gas region sampled: light blue for dust profiles extracted close to the water region $(\sim 2.9 \mu \mathrm{m})$, orange for the nitrile region $(\sim 3.0-3.1 \mu \mathrm{m})$, purple $(\sim 3.3 \mu \mathrm{m})$ and green $(\sim 3.6 \mu \mathrm{m})$ for the hydrocarbonstretch region, and dark blue for the $\mathrm{C}-\mathrm{O}$ and $\mathrm{C}-\mathrm{N}$ stretch region $(4.7-5.0 \mu \mathrm{m})$. Lower panels: comparison of the different gas profiles on the $2020 \mathrm{July} 20$. Here the same dust profiles as above are shown in black dotted lines, while the gas is in colored full lines. These plots show the difference in the shifts between water and the other molecules; $\mathrm{NH}_{2}$ is unique in showing two major peaks-one associated with the dust continuum and the other associated with primary molecular species (see text). Profiles for $\mathrm{H}_{2} \mathrm{O}, \mathrm{HCN}, \mathrm{C}_{2} \mathrm{H}_{6}$, and dust are compared in the rightmost lower panel.

dissociation product of solid-phase (semi-volatile) ammonium salts (Altwegg et al. 2016; Mumma et al. 2018, 2019; Altwegg et al. 2020; Poch et al. 2020). Ammoniated salts (e.g., $\left[\mathrm{NH}_{4}^{+}\right.$
]$\left.\left[\mathrm{CN}^{-}\right]\right)$can be produced at $10-15 \mathrm{~K}$ in laboratory simulations of ISM acid-base reactions (Gerakines et al. 2004; Bergner et al. 2016); once formed, such salts will survive until warmed 

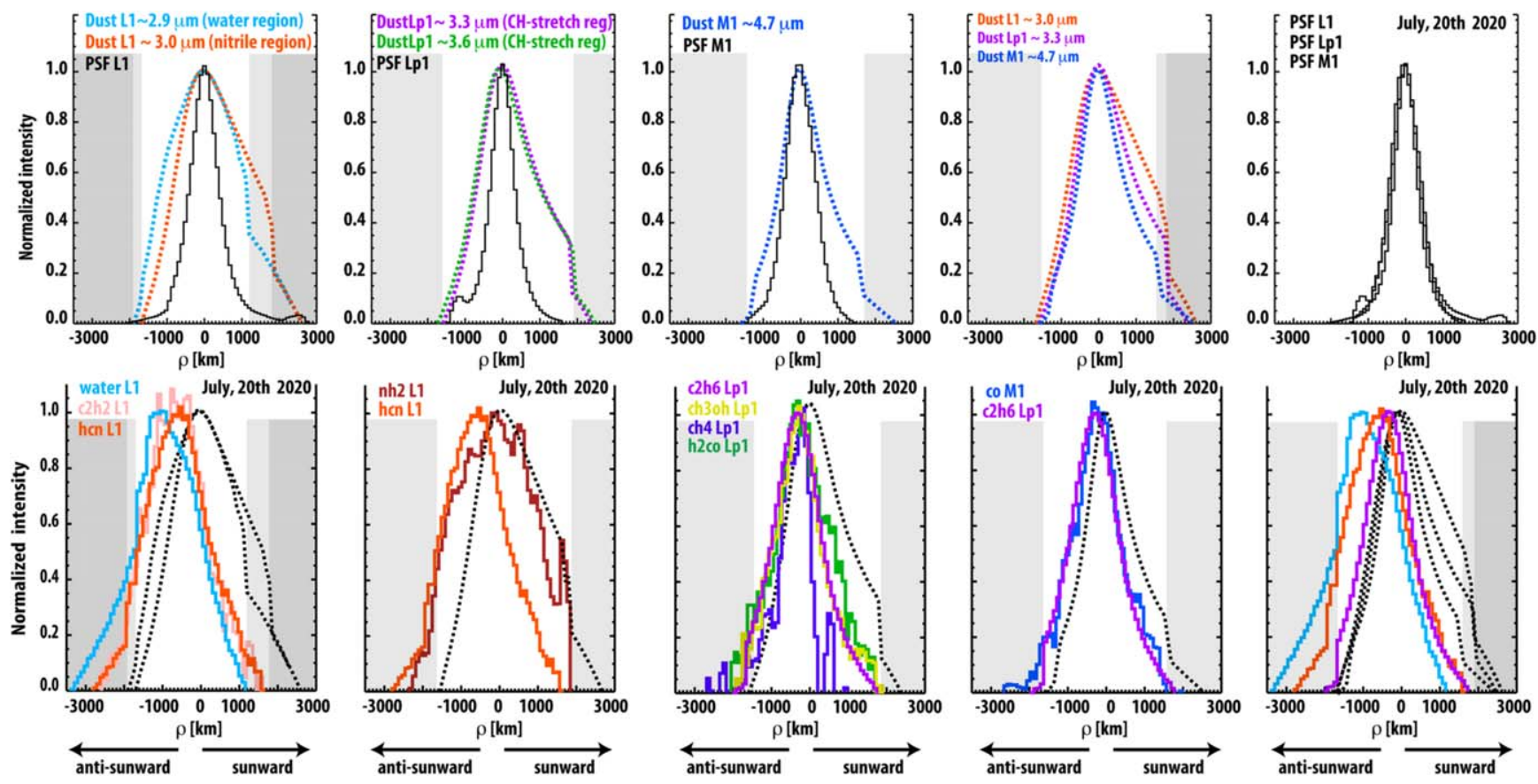

(b)

Figure 3. (Continued.)

to about $200 \mathrm{~K}$ and higher, meaning that the solid-phase salts now present in the cometary nucleus could have formed in the natal interstellar cloud core. Once ejected by a comet that passes very close to the Sun $(<1 \mathrm{au})$, this material could be heated to dissociation temperatures by solar radiation and it can dissociate into its constituent species (e.g., $\mathrm{HCN}, \mathrm{CN}, \mathrm{NH}_{3}$, and $\mathrm{NH}_{2}$ ); smaller grains would reach higher temperatures (superheat) and possibly reveal the presence of extended sources of gaseous products in cometary comae (Mumma et al. 2019). Ammonium salts have not been studied yet in a cometary context at near-IR wavelengths, where both solid-phase continuum features and gas-phase molecular emissions can be observed; a dedicated paper focused on investigations of the cometary continuum will address these issues.

A summary of measured relative pixel shifts for setting L1c/ iSHELL is reported in Table 2.

In setting Lp1c/iSHELL (3.3-3.5 $\mu \mathrm{m})$, spatial distributions of $\mathrm{C}_{2} \mathrm{H}_{6}, \mathrm{CH}_{3} \mathrm{OH}, \mathrm{CH}_{4}$, and $\mathrm{H}_{2} \mathrm{CO}$ are all shifted by 4 pixels, as are water and $\mathrm{CO}$ in the iSHELL/M1 setting $(4.5-5.2 \mu \mathrm{m})$ (Figure 3(b), third and fourth lower panels). A comparison of three molecules with different shifts (water 13 pixel shift, HCN 6 pixel shift, and $\mathrm{C}_{2} \mathrm{H}_{6} 4$ pixel shift) is shown in the fifth lowerright panel of Figure 3(b). This effect might be related to the fact that the detected gas came from the sublimation of an extended source of icy grains in the coma, most likely composed of water ice, which probably was dominating the activity in that region of the coma at that time, followed by the sublimation of the other organic ices mixed with the water ice. However, we cannot discard the fact that the presence of a spiral jet, composed of icy grains of different mixtures, could fit as another possible explanation. Our interpretation cannot quantitatively distinguish between either of those possible sources in explaining the observed shifts in the spatial profiles.

If we look at the top panels of Figure 3(b) indeed, we can investigate and interconnect spatial profile to the dust distribution. Dust profiles were extracted close to the bright molecular emission lines and used to evaluate the spatial profiles of gases. On July 20 we used three spectral settings: L1c, Lp1c, and M1, extracting information on the dust distribution spanning wavelengths from the 2.9 to the $4.7 \mu \mathrm{m}$ wavelengths region. It is possible to note that only the dust profile extracted close to the water emission lines in the $2.9 \mu \mathrm{m}$ region (first left top panel of Figure 3(b), dotted light-blue line) appears to be more extended, in the antisunward direction (negative $\rho$ ), compared with the dust profile extracted close to the nitrile emission lines in the 3.0-3.1 $\mu \mathrm{m}$ region (first left top panel of Figure 3(b), dotted orange line). The latter (still in the antisunward direction) instead is consistent with the dust profiles extracted close to molecular emission lines in the 3.3 and $3.6 \mu \mathrm{m}$ regions (fourth top panel of Figure 3(b), dotted purple and green lines) and consistent with those close to the molecular emission lines in the $4.7 \mu \mathrm{m}$ region (fourth top panel of Figure 3(b), dotted blue line). This might be qualitatively interpreted as a hint of icy grains ejected into the antisunward coma region, which reflect sunlight and that release water vapor there.

If we now look at the sunward direction (positive $\rho$ ), all dust profiles appear to be enhanced if compared to the stellar profile. This is likely associated with thermal emission from refractory grains in the sunward-facing hemisphere of the coma. These profiles exhibit a very interesting trend with wavelength. At shorter wavelengths between 2.9 and $3.3 \mu \mathrm{m}$, the continuum flux density, which is composed of both the reflected sunlight and the thermal emission components, is more extended than in the $\mathrm{M}$ band $(\sim 5 \mu \mathrm{m})$ where the thermal emission component dominates the continuum. This is probably why we notice a steeper slope at $5 \mu \mathrm{m}$ than at $\sim 3 \mu \mathrm{m}$ in the dust spatial distribution (see fourth top panel of Figure 3(b)).

Starting with July 31 and continuing on the other observing dates, in 2020 August the molecular spatial profiles appeared to follow the dust spatial profiles with no spatial shift. Both the gas 
and the dust distributions looked symmetric. Interestingly, $\mathrm{NH}_{2}$ kept showing an extended profile in the antisunward direction, again associated with the possible presence of two components.

Sharp drops in the profiles indicate the end of the combined sections of the spectral order. The iSHELL slit length is $15^{\prime \prime}$, shorter than NIRSPEC's slit that has a length of $24^{\prime \prime}$. When nodding on slit with iSHELL, the A- and B-beam positions are symmetric relative to the slit center and are positioned halfway from the center to the end of the slit. So, keeping this in mind, our technique of combining the $\mathrm{A}$ and the $\mathrm{B}$ beams as ABBA sets reduces to practically half the length $\left(\sim 7^{\prime \prime}\right)$ the range over which we can study the molecular spatial outgassing distributions. Due to the large shifts between the gas and the dust, sometimes the profiles appear to be truncated in the ABBA extracts. Moreover, guiding on the comet during daytime might not be perfectly stable, causing the object to move on the slit and thus slightly reducing the slit length over which we can perform the spatial analysis. As mentioned at the beginning of this section, in Figures 3(a) and (b) we marked in gray the regions outside the "good" ABBA combined area; such regions are discarded in our analysis.

Comet NEOWISE had a very large coma, and due to the short iSHELL slit, a possible impact of oversubtraction of the cometary signal in the spatial region between the A and the $\mathrm{B}$ beams requires discussion. In principle, oversubtraction between the two beams is an effect that occurs in all cometary observations. However, it is important to consider that the relative impact of this effect is typically proportional to the cometary nucleocentric distance as $\sim 1 / \rho$.

As already mentioned, the iSHELL slit length is $15^{\prime \prime}$ and the nodding distance between the $\mathrm{A}$ and the $\mathrm{B}$ beams is then $\sim 7^{\prime \prime}$. If we consider comet NEOWISE spatial information as reported in Table 2, on average, at such geocentric distances, $7^{\prime \prime}$ would correspond to $\sim 40$ pixels, and it would be equivalent to $\sim 4000$ $\mathrm{km}$ between the beams. At such large nucleocentric distances, the signal from the other beam would be greatly reduced, so its effect of oversubtracting would have a marginal impact on the extracted production rates. An impact on the profile analysis might still be present, yet it would not be substantial.

A way to solve this would be to extract the A and the B beams alone and analyze them separately; however, the advantages of performing ABBA beam subtraction, with their consecutive combination, enables optimum sky cancellation, which would not be possible when treating each beam independently. Although we see the value of treating each beam independently, we decided to perform our standard ABBA reduction technique because, when observing during daytime, sky removal can be a much greater issue than oversubtraction.

\subsubsection{Slit-loss Correction}

As mentioned at the beginning of the previous section, fully calibrated "centered" 8 cometary spectra were extracted over defined rectangular apertures of sizes that depend on the adopted slit width as reported (see Table 2). For each molecular species, the "centered" spectrum was extracted by centering the reference central pixel on the row containing the peak emission intensity for such molecule. From each specific molecular

\footnotetext{
8 To be clear, we call "centered" the spectrum extracted with the reference central pixel taken from the observed different molecular offset positions. When no gas shifts are present, the "centered" spectrum is the standard "nucleus-centered" spectrum, extracted from the reference central pixel position (offset $=0$ ).
}

spatial profile, an appropriate slit-loss correction was evaluated through the "Q-curve methodology" (Xie \& Mumma 1996; Dello Russo et al. 1998; Villanueva et al. 2011b), by retrieving the growth factor $\left(\mathrm{Q}_{\text {scale }}\right)$. The growth factor is a correction factor that primarily accounts for the loss of flux due to the atmospheric seeing but is also for possible guiding drifts during the acquisition of ABBA nodding sequences. Conceptually, this methodology is trivially valid for an isotropic coma, but it is also valid for an axisymmetric coma as first shown by Xie \& Mumma (1996). When asymmetric profiles are present, the treatment of the Q-curve methodology is slightly more complex (see details in Villanueva et al. 2011b). Usually, we compute the $\mathrm{Q}_{\text {scale }}$ on the left and the right parts of the profile and we then symmetrize them (take the weighted mean) at each slant distance from the nucleus. The mean values increase with slant distance until a terminal value is reached at some distance from the nucleus, and this is taken to represent the global production. Gas spatial profiles in comet NEOWISE did not appear to be strongly asymmetric but rather to be shifted into the antisunward direction, but we applied this method anyway to correct for even small asymmetries. In Table 3 we summarize the measured $\mathrm{Q}_{\text {scale }}$ for each molecule. The errors in the $\mathrm{Q}_{\text {scale }}$ presented in Table 3 include the systematic deviations of the profiles with respect to an isotropic profile.

For iSHELL observations, when the measured scale factors for molecules sampled in a given spectral setting were consistent within their confidence limits, we decided to adopt a weighted mean $\mathrm{Q}_{\text {scale }}$ value for such a setting. When not consistent within confidence limits, we adopted the measured $\mathrm{Q}_{\text {scale }}$ for that specific molecular species (e.g., $\mathrm{H}_{2} \mathrm{CO}$ ). Because we observed with a single setting (KL1) for NIRSPEC investigations, we adopted the $\mathrm{Q}_{\text {scale }}$ measured for water when the spatial profile of a molecular species was too noisy. Adopted $\mathrm{Q}_{\text {scale }}$ values are also shown in Table 4 .

\subsection{Rotational Analysis and Molecular Production Rates}

"Centered" (or "nucleus-centered" when offset is zero) production rates $\left(\mathrm{Q}_{\text {nucl }}\right)$ are obtained from the calibrated "centered" extracted cometary spectra (at different offset positions), through the well-documented correlation and rotational analysis methods (DiSanti et al. 2001; Bonev 2005; Villanueva et al. 2011a). Both methods allow to retrieve accurate rotational temperatures $T_{\text {rot }}$, which are essential for extracting nucleus-centered production rates $\mathrm{Q}_{\text {nucl }}{ }^{9}$

The correlation analysis method retrieves rotational temperatures $T_{\text {rot }}$ from the evaluation of correlation coefficients between the observed and the synthetic spectrum. $T_{\text {rot }}$ is defined as the temperature that produces the best correlation (see Figure 4). The rotational analysis method instead retrieves $T_{\text {rot }}$ considering the relationship between the ratio of the observed and modeled line fluxes. The observed line fluxes are corrected for terrestrial transmittance at the Doppler-shifted line frequency) while the $g$-factors (photons $/ \mathrm{mol} / \mathrm{s}$ ) are predicted using quantum mechanical fluorescence models developed by the Goddard team (DiSanti et al. 2006; Villanueva et al. 2011a, 2012a, 2012b), including full cascade formalisms resulting from pumping transitions that originate in levels of the ground vibrational state $\left(E_{\text {rot }}\right)$ whose populations are

\footnotetext{
9 We keep the standard definition of $Q_{\text {nucl }}$ to avoid confusion. However, centered production rates are computed from the extracted spectra, centered at different offset positions. When the offset is zero (no gas shift) then the centered production rates correspond to the nucleus-centered production rates.
} 
Table 3

Summary of Growth Factors

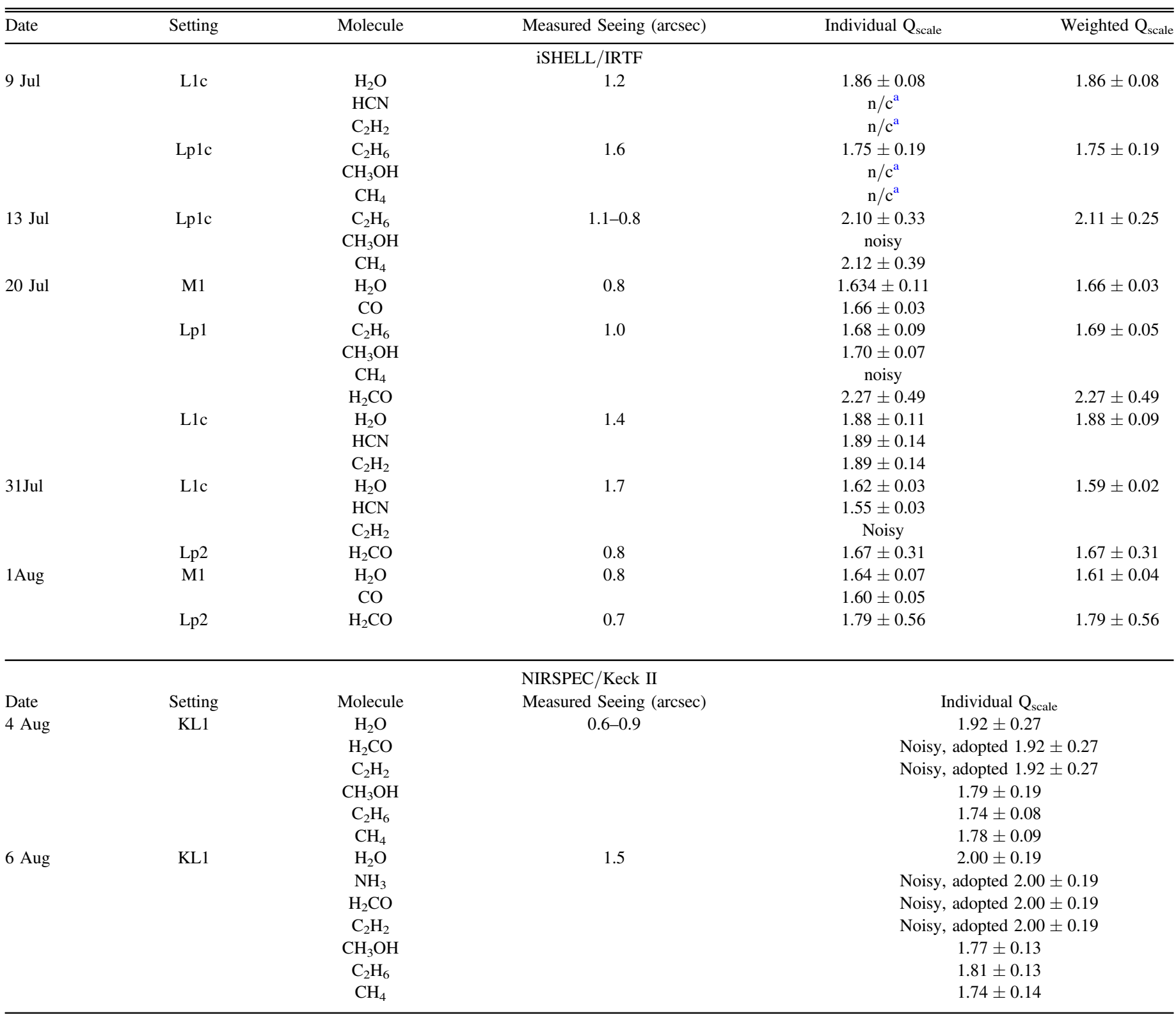

Note.

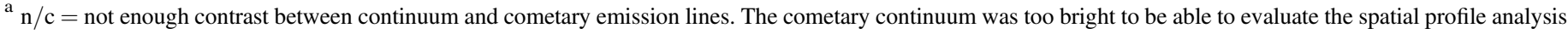
on the cometary emission lines.

calculated for a specific $T_{\text {rot }}(\mathrm{K})$. Values of $T_{\text {rot }}$ are varied until the slope of the fitted lines is zero (see Figure 4). The $\pm 1 \sigma$ uncertainties in $T_{\text {rot }}$ are computed from the statistical errors of the rotational analysis method. We selected the higher uncertainty of the stochastic error (which describes the uncertainty produced by photon noise) and the variance error (standard error, which describes the spread of the derived quantities around the slope line). These methodologies are described in detail in earlier works (Faggi et al. 2018, 2019).

In Table 4, a summary of the retrieved molecular production rates is reported. The total production rate $\left(\mathrm{Q}_{\text {tot }}\right)$ for each species is obtained by applying to each centered (nucleuscentered) production rate the slit-loss correction, as follows: $\mathrm{Q}_{\text {tot }}=\mathrm{Q}_{\text {nucl }} \times \mathrm{Q}_{\text {scale }}($ see Table 4$)$.
In Figure 4 we show examples of the rotational analysis method for multiple molecules across different dates and the results of both the rotational and correlation analysis methods in finding the best rotational temperature $T_{\text {rot }}$.

\subsubsection{Water Production Rate}

Measured centered (nucleus-centered) and total water production rates are reported in Table 4 and graphed in Figure 5. We detected many water emission lines, around $2.9 \mu \mathrm{m}$, throughout the observing campaign. On July 9 in the L1c setting of iSHELL, 52 water rovibrational lines were detected; on July 20 we detected 78 lines; and on July 31, 133 water lines were detected. On August 1 water measurements were extracted from the M1 setting of iSHELL, where we detected 21 rovibrational lines near 
Table 4

Retrieved Rotational Temperatures, Production Rates, and Mixing Ratios for Primary Volatiles in C/2020 F3 NEOWISE

\begin{tabular}{|c|c|c|c|c|c|c|c|}
\hline Date & Spectral Setting & Molecule & $T_{\text {rot }}^{\mathrm{a}}(\mathrm{K})$ & $\mathrm{Q}_{\text {nucl }}{ }^{\mathrm{b}}\left(10^{26} \mathrm{~s}^{-1}\right)$ & $\mathrm{Q}_{\text {scale }}$ & $Q_{\text {tot }}\left(10^{26} \mathrm{~s}^{-1}\right)$ & Mixing ratio ${ }^{c}(\%)$ \\
\hline & & & & iSHELL/IRTF & & & \\
\hline \multirow[t]{7}{*}{$9 \mathrm{Jul}$} & L1c & $\mathrm{H}_{2} \mathrm{O}$ & $155 \pm 4$ & $2570.9 \pm 41.4$ & $1.86 \pm 0.08$ & $4777.6 \pm 213.1$ & 100 \\
\hline & & $\mathrm{HCN}$ & $87 \pm 18$ & $7.90 \pm 0.80$ & & $14.67 \pm 1.61$ & $0.31 \pm 0.04$ \\
\hline & & $\mathrm{C}_{2} \mathrm{H}_{2}$ & (155) & $7.69 \pm 0.95$ & & $14.28 \pm 1.87$ & $0.30 \pm 0.04$ \\
\hline & & $\mathrm{NH}_{3}{ }^{\mathrm{d}}$ & (155) & $<20.98$ & & $<38.93$ & $<0.77$ \\
\hline & Lp1c & $\mathrm{C}_{2} \mathrm{H}_{6}$ & (155) & $6.74 \pm 0.44$ & $1.75 \pm 0.19$ & $11.76 \pm 1.51$ & $0.24 \pm 0.03$ \\
\hline & & $\mathrm{CH}_{3} \mathrm{OH}$ & (155) & $<8.70$ & & $<15.18$ & $<0.32$ \\
\hline & & $\mathrm{CH}_{4}$ & $(155)$ & $7.67 \pm 2.78$ & & $13.38 \pm 5.07$ & $0.27 \pm 0.10$ \\
\hline \multirow[t]{3}{*}{$14 \mathrm{Jul}$} & Lp1c & $\mathrm{C}_{2} \mathrm{H}_{6}$ & $146 \pm 19$ & $6.93 \pm 0.24$ & $2.11 \pm 0.25$ & $14.60 \pm 1.82$ & $0.32 \pm 0.01^{\mathrm{e}}$ \\
\hline & & $\mathrm{CH}_{3} \mathrm{OH}$ & (146) & $10.29 \pm 1.29$ & & $21.68 \pm 3.75$ & $0.47 \pm 0.05^{\mathrm{e}}$ \\
\hline & & $\mathrm{CH}_{4}$ & $122 \pm 24$ & $7.08 \pm 0.86$ & & $14.91 \pm 2.54$ & $0.32 \pm 0.04^{\mathrm{e}}$ \\
\hline \multirow[t]{11}{*}{$20 \mathrm{Jul}$} & L1c & $\mathrm{H}_{2} \mathrm{O}$ & $129 \pm 4$ & $2232.4 \pm 41.8$ & $1.88 \pm 0.09$ & $4205.4 \pm 213.7$ & \\
\hline & M1 & & $111 \pm 4$ & $2728.7 \pm 77.8$ & $1.66 \pm 0.03$ & $4521.4 \pm 154.2$ & \\
\hline & $\mathrm{L} 1 \mathrm{c}+\mathrm{M} 1$ & & $128 \pm 3$ & $2242.9 \pm 37.6$ & & $4413.2 \pm 125.1^{\mathrm{f}}$ & 100 \\
\hline & M1 & $\mathrm{CO}$ & $123 \pm 11$ & $49.94 \pm 2.24$ & $1.66 \pm 0.03$ & $82.76 \pm 4.02$ & $1.97 \pm 0.14$ \\
\hline & Lp1 & $\mathrm{C}_{2} \mathrm{H}_{6}$ & $131 \pm 7$ & $24.26 \pm 0.39$ & $1.69 \pm 0.05$ & $41.01 \pm 1.45$ & $0.98 \pm 0.06$ \\
\hline & & $\mathrm{CH}_{3} \mathrm{OH}$ & $102 \pm 7$ & $53.72 \pm 1.58$ & & $90.79 \pm 3.91$ & $2.16 \pm 0.14$ \\
\hline & & $\mathrm{CH}_{4}$ & (130) & $35.79 \pm 6.96$ & & $60.48 \pm 11.92$ & $1.44 \pm 0.29$ \\
\hline & & $\mathrm{H}_{2} \mathrm{CO}$ & (130) & $10.02 \pm 0.93$ & $2.27 \pm 0.49$ & $22.79 \pm 5.33$ & $0.54 \pm 0.13$ \\
\hline & L1c & $\mathrm{HCN}$ & $85 \pm 7$ & $5.21 \pm 0.25$ & $1.88 \pm 0.09$ & $9.98 \pm 0.66$ & $0.24 \pm 0.02$ \\
\hline & & $\mathrm{C}_{2} \mathrm{H}_{2}$ & $(130)$ & $62.42 \pm 0.31$ & & $11.76 \pm 0.80$ & $0.28 \pm 0.02$ \\
\hline & & $\mathrm{NH}_{3}{ }^{\mathrm{d}}$ & $(130)$ & $<26.97$ & & $<50.81$ & $<1.15$ \\
\hline \multirow[t]{5}{*}{ 31Jul } & L1c & $\mathrm{H}_{2} \mathrm{O}$ & $88 \pm 3$ & $1005.8 \pm 30.4$ & $1.59 \pm 0.02$ & $1596.1 \pm 52.2$ & 100 \\
\hline & & $\mathrm{HCN}$ & $75 \pm 2$ & $2.27 \pm 0.04$ & & $3.60 \pm 0.07$ & $0.23 \pm 0.01$ \\
\hline & & $\mathrm{NH}_{3}{ }^{\mathrm{d}}$ & $(90)$ & $7.34 \pm 0.10$ & & $11.65 \pm 1.57$ & $0.73 \pm 0.10$ \\
\hline & & $\mathrm{C}_{2} \mathrm{H}_{2}$ & $89 \pm 5$ & $1.65 \pm 0.06$ & & $26.18 \pm 0.94$ & $0.16 \pm 0.01$ \\
\hline & Lp2 & $\mathrm{H}_{2} \mathrm{CO}$ & $108 \pm 7$ & $1.96 \pm 0.08$ & $1.67 \pm 0.31$ & $3.27 \pm 0.62$ & $0.21 \pm 0.04$ \\
\hline \multirow[t]{4}{*}{ 1Aug } & M1 & $\mathrm{H}_{2} \mathrm{O}$ & $66 \pm 9$ & $729.4 \pm 37.4$ & $1.61 \pm 0.04$ & $1176.5 \pm 767.4$ & 100 \\
\hline & & $\mathrm{CO}$ & $84 \pm 3$ & $18.57 \pm 0.39$ & & $29.95 \pm 0.98$ & $2.35 \pm 0.13$ \\
\hline & Lp2 & $\mathrm{H}_{2} \mathrm{CO}$ & $111 \pm 7$ & $1.71 \pm 0.07$ & $1.79 \pm 0.56$ & $3.08 \pm 0.96$ & $0.26 \pm 0.08$ \\
\hline & & & & NIRSPEC/Keck II & & & \\
\hline \multirow[t]{6}{*}{ 4Aug } & KL1 & $\mathrm{H}_{2} \mathrm{O}$ & $73 \pm 7$ & $546.6 \pm 35.1$ & $1.92 \pm 0.27$ & $1048.9 \pm 1597.6$ & 100 \\
\hline & & $\mathrm{H}_{2} \mathrm{CO}$ & $(75)$ & $<0.78$ & $1.92 \pm 0.27$ & $<1.49$ & $<0.14$ \\
\hline & & $\mathrm{CH}_{3} \mathrm{OH}$ & $85 \pm 6$ & $19.06 \pm 0.49$ & $1.79 \pm 0.19$ & $34.12 \pm 3.73$ & $3.25 \pm 0.61$ \\
\hline & & $\mathrm{C}_{2} \mathrm{H}_{6}$ & $88 \pm 5$ & $10.46 \pm 0.20$ & $1.74 \pm 0.08$ & $18.19 \pm 0.90$ & $1.73 \pm 0.28$ \\
\hline & & $\mathrm{C}_{2} \mathrm{H}_{2}$ & $(75)$ & $1.10 \pm 0.28$ & $1.92 \pm 0.27$ & $2.11 \pm 0.62$ & $0.20 \pm 0.07$ \\
\hline & & $\mathrm{CH}_{4}$ & $(75)$ & $13.20 \pm 1.36$ & $1.78 \pm 0.09$ & $23.49 \pm 3.12$ & $2.24 \pm 0.45$ \\
\hline \multirow[t]{7}{*}{ 6Aug } & KL1 & $\mathrm{H}_{2} \mathrm{O}$ & $73 \pm 3$ & $737.5 \pm 37.5$ & $2.00 \pm 0.19$ & $1475.0 \pm 158.9$ & 100 \\
\hline & & $\mathrm{NH}_{3}$ & $(75)$ & $6.79 \pm 1.03$ & $2.00 \pm 0.19$ & $13.58 \pm 2.43$ & $0.92 \pm 0.19$ \\
\hline & & $\mathrm{H}_{2} \mathrm{CO}$ & $(75)$ & $<1.19$ & $2.00 \pm 0.19$ & $<2.39$ & $<0.16$ \\
\hline & & $\mathrm{CH}_{3} \mathrm{OH}$ & $61 \pm 3$ & $17.42 \pm 0.41$ & $1.77 \pm 0.13$ & $30.83 \pm 2.38$ & $2.09 \pm 0.28$ \\
\hline & & $\mathrm{C}_{2} \mathrm{H}_{6}$ & $101 \pm 12$ & $10.70 \pm 0.32$ & $1.81 \pm 0.13$ & $19.28 \pm 1.50$ & $1.31 \pm 0.17$ \\
\hline & & $\mathrm{C}_{2} \mathrm{H}_{2}$ & $109 \pm 14$ & $1.63 \pm 0.13$ & $2.00 \pm 0.19$ & $3.27 \pm 0.40$ & $0.22 \pm 0.04$ \\
\hline & & $\mathrm{CH}_{4}$ & $90 \pm 13$ & $15.82 \pm 1.03$ & $1.74 \pm 0.14$ & $27.53 \pm 2.85$ & $1.87 \pm 0.28$ \\
\hline
\end{tabular}

Notes.

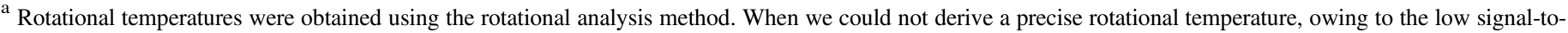

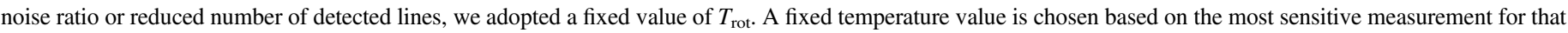

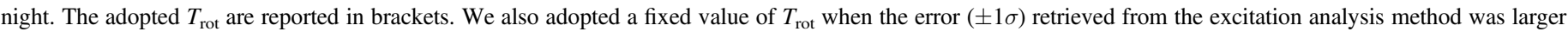
than $30 \mathrm{~K}$.

${ }^{\mathrm{b}}$ Detections lower than $3 \sigma$ are reported here as $(3 \sigma)$ upper limits. Their measured levels and confidence limits are given in the text.

c Mixing ratios relative to measured water are reported.

${ }^{\mathrm{d}}$ Measurements of ammonia are usually performed on one or two very faint spectral lines, so we adopted the water $T_{\text {rot }}$.

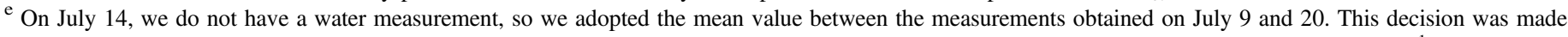
because the water production rate between those dates appeared stable. The assumed value from which we computed MR is $(45.9 \pm 1.7) \mathrm{e}+28 \mathrm{~s}^{-1}$.

${ }^{\mathrm{f}}$ Weighted mean of the total water production rate in the setting L1c and M1.

$4.7 \mu \mathrm{m}$. On August 4 and 6,16 and 39 water lines were detected near $2.9 \mu \mathrm{m}$ in the KL1 setting of NIRSPEC, respectively.

On July 14, due to bad weather, we missed investigations of the L1c setting and therefore we do not have a measurement of the absolute water production rate. Based on the near stability of the water measurements we obtained between July 9 and 20, with $\mathrm{Q}\left(\mathrm{H}_{2} \mathrm{O}\right)_{\text {tot }}=(4.778 \pm 0.213) \times 10^{29}\left(\mathrm{~s}^{-1}\right)$ and $\mathrm{Q}\left(\mathrm{H}_{2} \mathrm{O}\right)_{\text {tot }}=$ $(4.413 \pm 0.125) \times 10^{29}\left(\mathrm{~s}^{-1}\right)$, respectively, we decided to adopt the average value between such measurements $\mathrm{Q}\left(\mathrm{H}_{2} \mathrm{O}\right)_{\text {tot }}=$ $(4.595 \pm 0.169) \times 10^{29}\left(\mathrm{~s}^{-1}\right)$ for July 14 . We computed molecular abundances relative to water on July 14 using this mean. 

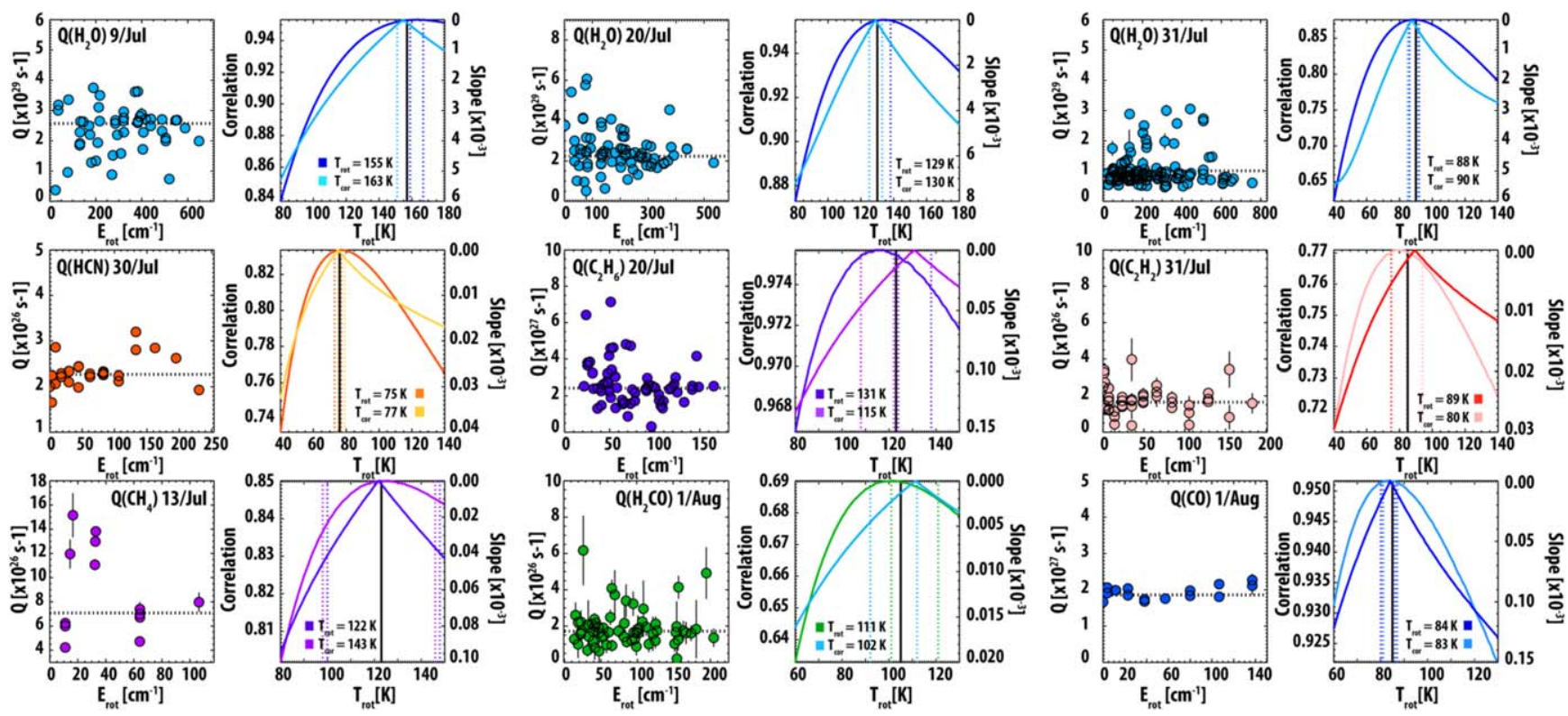

Figure 4. Rotational temperature and production rate analyses for water and organic trace gases in C/2020 F3 NEOWISE on multiple dates. In the temperature charts, distinct colors represent the two methods while the vertical colored dotted lines represent the $\pm 1 \sigma$ uncertainties on $T_{\text {rot }}$.

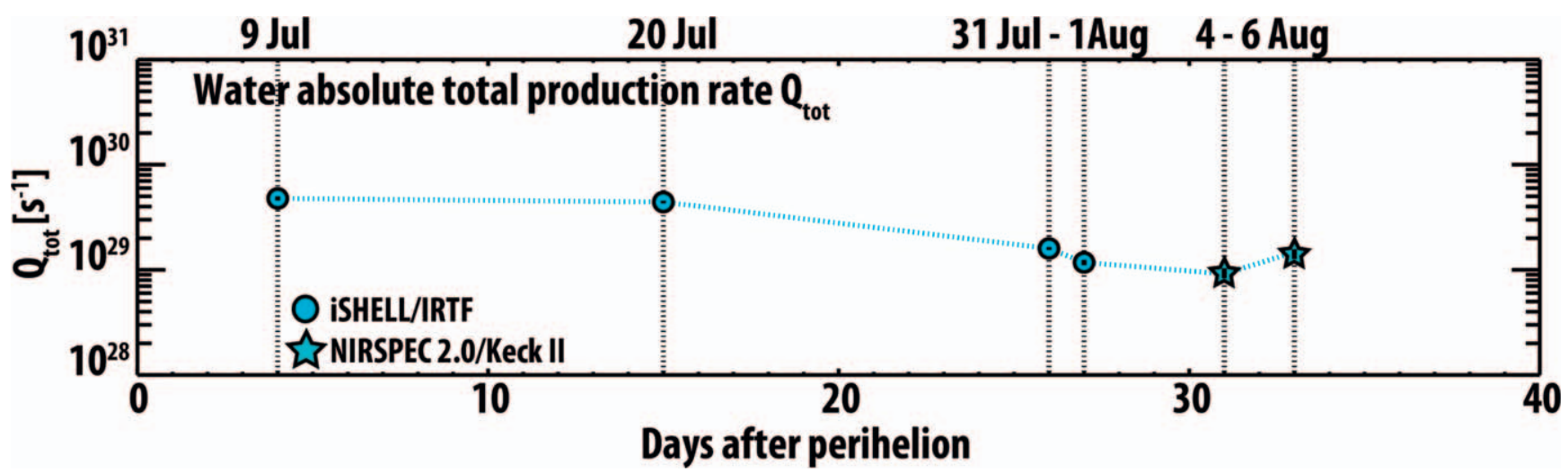

Figure 5. Temporal variability of the global water production rate $\left(\mathrm{s}^{-1}\right)$ in comet NEOWISE. Circles refer to IRTF observations while stars refer to NIRSPEC observations.

As discussed in the spatial profile analysis, two possible sources may have contributed to water in the cometary coma: the direct outgassing from the nucleus, and the release associated with sublimation of icy grains in the cometary coma within the sampled field of view, which encompassed a $\sim \pm 3000(\mathrm{~km})^{10}$ projected slant distance along the sunward and antisunward directions. The hypothesis of two possible sources of water production was supported by both the large shifts observed between the gas- and dust-profile peaks and the enhancements of the gas profile and the dust distribution in the antisunward direction ((see Figure 3(b), first top and first bottom panels on the left). The observed large gas-to-dust shift on July 20 (13 pixels, which is $\sim 1113 \mathrm{~km}$ ) might be explained by the large amount of water sublimating from icy grains, which dominated outflowing water vapor released directly from the nucleus. As observed in comet Boattini (Villanueva et al. 2011b) and comet Garradd (Villanueva et al. 2012c), nearly pure water-ice grains might be invoked in this scenario because they would sublime at a slower pace compared with dusty-icy grains. Nearly pure ice grains lack the dark refractory material

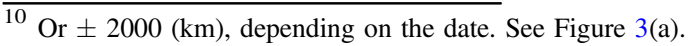

component that would more effectively absorb the solar radiation and lead to a higher grain temperature. The excess water produced by icy grains can be investigated by comparing the water molecular spatial profiles observed on July 20 with those on July 31 where the water spatial profiles followed the dust distribution, and no spatial shifts are present. Indeed, on the last four observing dates (July 31 and August 1, 4, and 6) the profiles appeared symmetric.

If on July 20 the measured total water production rate came from the sum of two components (the water outgassing from the nucleus $\left(\mathrm{Q}_{N}\right)$ and the water outgassing from the extended icy grains $\left.\left(\mathrm{Q}_{\text {ext }}\right)\right)$, we could then write the measured water production rate as $\mathrm{Q}\left(\mathrm{H}_{2} \mathrm{O}\right)=\left(\mathrm{Q}_{N}+\mathrm{Q}_{\mathrm{ext}}\right)=4.205 \times 10^{29}$ $\left(\mathrm{s}^{-1}\right)$. If now we assume that on July 31 the measured total water production rate was instead dominated by the nucleus outgassing alone: $\mathrm{Q}\left(\mathrm{H}_{2} \mathrm{O}\right)=\left(\mathrm{Q}_{N}\right)=1.596 \times 10^{29}\left(\mathrm{~s}^{-1}\right)$, and we assume that the water outgassed from the nucleus did not change significantly between the observed dates (as confirmed by the measurements among August observing runs), we can estimate the "excess water production" produced by icy grains $\left(\mathrm{Q}_{\mathrm{ext}}\right)$ to be $\sim 1.63$ times the outgassing from the nuclear component $\left(\mathrm{Q}_{\mathrm{ext}}=2.609 \times 10^{29}\left(\mathrm{~s}^{-1}\right)\right)$. The ratios $\mathrm{Q}_{N} /\left(\mathrm{Q}_{N}+\right.$ 


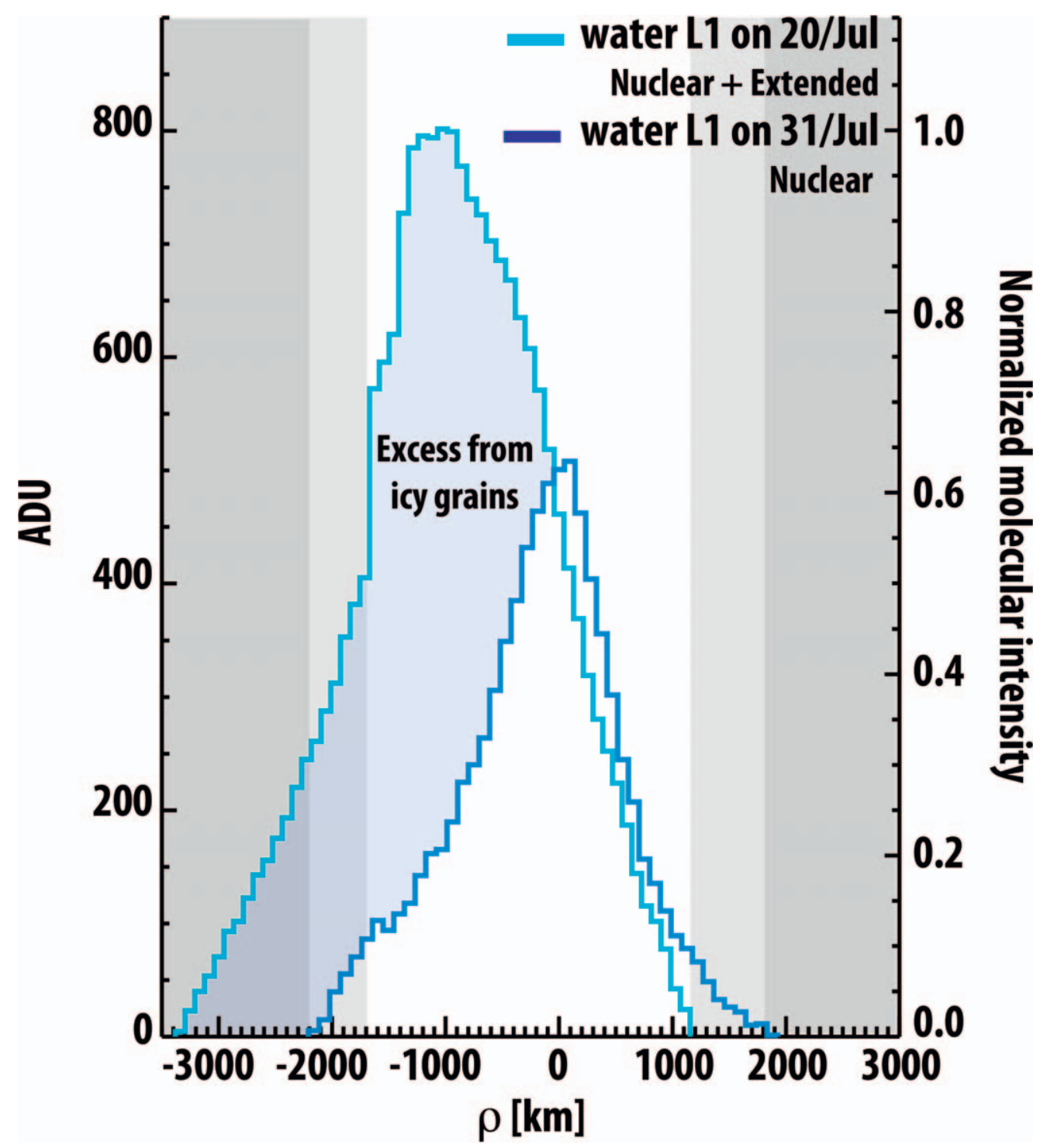

Figure 6. Comparison between spatial profiles of gaseous water extracted 2020 July 20 and July 31 . The left scale quantifies the measured profiles in ADU, while the right scale compares normalized profiles. On the first date the water profile peaks at $\sim-1113 \mathrm{~km}$, in the antisunward direction. Water excess at such a distance can likely be associated with the sublimation of micron-sized nearly pure water ice grains after their release from the nucleus.

$\left.\mathrm{Q}_{\mathrm{ext}}\right)=0.38$ and $\mathrm{Q}_{\text {ext }} /\left(\mathrm{Q}_{N}+\mathrm{Q}_{\mathrm{ext}}\right)=0.62$ can roughly identify the fraction of water production coming directly from the nucleus $(38 \%)$ and the fraction from the extended source of icy grains $(62 \%)$.

In Figure 6 we show a comparison between the molecular spatial profiles for water extracted on July 20 and on July 31 . In the graphic, we compare the area below the two profiles, and we estimate the excess produced by the sublimation of icy grains as the difference between the two areas, which are proportional to the integrated flux density. The area below the light-blue curve (July 20) is $A_{N+\text { ext }}=19768.8$ ADU while that one below the dark blue curve (July 31 ) is $A_{N}=8282.61 \mathrm{ADU}$. The ratios obtained from the areas provide a second metric for identifying the two components: $A_{N} / A_{N+\mathrm{ext}}=0.42(42 \%)$ and $A_{\text {ext }} / A_{N+\text { ext }}=0.58(58 \%)$, which are close to the related percentages estimated from the production rates. If we now consider the gas-to-dust shift on July 20 (13 pixels, or $\sim 1113$ $\mathrm{km}$ ), we could try to explain the water excess (peak) at such a distance by the sublimation of small-sized perhaps nearly pure water ice grains after their release from the nucleus. In this scenario, larger grains in the coma would show velocities that are slower than the gas velocity (i.e., $v_{\text {grain }}<v_{\text {gas }}=0.8$ $R_{H}^{-0.5}=1.07 \mathrm{~km} \mathrm{~s}^{-1}$ ), because the outflowing gas cannot accelerate them to the terminal gas outflow velocity, so if we assume $v_{\text {icy-grains }}=v_{\text {gas }}$, we can then estimate a grain lifetime about $\sim 1040 \mathrm{~s}$, which (following Beer et al. 2006) will lead us to a modeled nearly pure-ice grain size of $\sim 1 \mu \mathrm{m}$ at heliocentric distances between 0.48 and $0.68 \mathrm{au}$. Importantly, this suggested possible lifetime of $\sim 1040 \mathrm{~s}$ should be taken as a qualitative guide of the magnitude of the effect. The lifetime of dust grains is highly dependent on the assumed grain-size and water-ice abundance.

Water production rates in comet $\mathrm{C} / 2020 \mathrm{~F} 3$ NEOWISE, measured in the near-IR and presented here, can be compared with measurements obtained from the SWAN instrument on board the SOHO space observatory, which followed the comet from 2020 May to September (Combi et al. 2021). If the cometary hydrogen coma is formed mainly by photodissociation of water into $\mathrm{H}$ and $\mathrm{OH}$ and then of $\mathrm{OH}$ into $\mathrm{O}$ and $\mathrm{H}$ atoms, the analysis of the hydrogen Ly $\alpha$ emissions (detected in 
the SWAN images) can provide water production rate measurements by modeling the photochemical kinetics and the thermalization of hot $\mathrm{H}$ atoms (Bertaux et al. 1999; Mäkinen \& Combi 2005). Comet NEOWISE was followed over 74 days from the middle of 2020 May to early 2020 September, with a data gap from 2020 June 18 to July 7 when the comet was too close to the Sun for SOHO observations. Water measurements $\left(\mathrm{Q}\left(\mathrm{H}_{2} \mathrm{O}\right)_{\mathrm{Ly \alpha}}\right)$ reported for dates close to our observations are the following: $5.27 \times 10^{30}\left(\mathrm{~s}^{-1}\right)$ at $\sim 0.34$ au (5.2 days after perihelion), $1.66 \times 10^{30}\left(\mathrm{~s}^{-1}\right)$ at $\sim$ $0.43 \mathrm{au}$ (10.1 days after perihelion), $1.10 \times 10^{30}\left(\mathrm{~s}^{-1}\right)$ at $\sim$ $0.54 \mathrm{au}$ (15.4 days after perihelion), $2.16 \times 10^{29}\left(\mathrm{~s}^{-1}\right)$ at 0.81 au (28.0 days after perihelion), and $1.95 \times 10^{29}\left(\mathrm{~s}^{-1}\right)$ at $0.84 \mathrm{au}$ (29.1 days after perihelion; Combi et al. 2021). Between $\sim 0.7$ and $0.8 \mathrm{au}, \mathrm{Ly} \alpha$ and near-IR water measurements mostly agree. At $\sim 0.34 \mathrm{au}, \sim 0.43 \mathrm{au}$, and $\sim 0.54$ au SWAN water measurements are almost one order of magnitude higher than near-IR direct water measurements; this might be explained by these considerations: we are sampling very different fields of view and the SWAN's much larger field of view can capture larger coma, thus a larger portion of the ejected icy-grain halo in it. An alternative interpretation involves the assumption that the detected cometary hydrogen is not formed mainly by photodissociation of water, but it might have different origins. For example, the destruction of very small-size solid-phase material, ejected in the coma together with the icy grains, might be responsible for the release of vast amounts of the excess hydrogen seen by SOHO and be interpreted as higher inferred water production rates, relative to the near-IR direct water measurements.

Qualitatively, we could think that possible origins of this excess hydrogen might be associated with the presence of ammonium salts and/or complex organic molecules. As an example, the complex salts ammonium formate $\left[\mathrm{NH}_{4}\right]^{+}[\mathrm{OOCH}]^{-}$, ammonium acetate $\left(\left[\mathrm{NH}_{4}\right]^{+}\left[\mathrm{COOCH}_{3}\right]^{-}\right.$, and ammonium glycinate $\left(\left[\mathrm{NH}_{4}\right]^{+}\left[\mathrm{CH}_{2} \mathrm{NH}_{2} \mathrm{COO}\right]^{-}\right.$contain five, seven, and eight hydrogen atoms per molecule, respectively. Ammonium salts sublimate at high temperatures and are expected to inflate the inferred $\operatorname{Ly} \alpha$ water measurements rapidly with decreasing heliocentric distance. However, complex organic molecules have more hydrogen atoms than ammonium salts, so our interpretation is qualitative while quantitative considerations are necessary to properly characterize different sources.

\subsubsection{Molecular Mixing Ratios Relative to Water}

The chemical inventory of comets is very rich and diverse. At various formative stages, interstellar-ice-like, cold grain surface chemistry-like, and warm disk photosphere-like processing could have affected the cometary chemical composition (Tielens 2013). Molecular abundance ratios detected in cometary nuclei may retain the record of the processing experienced in primitive stages of the protoplanetary disk, where the local temperatures and radiation fields defined conditions that influenced the ultimate chemical complexity of the emerging organic mix.

Usually and historically, water provides a natural "baseline" for comparison with other abundances. Molecular abundance ratios (or mixing ratios) are computed as the ratio between the measured molecular production rates and the water production rate, retrieved in a self-consistent fashion. The importance of performing simultaneous measurements of water and organic species rests on the fact that multiple sources of systematic uncertainty are in this way removed. Water outgassing dominates the activity of most active comets within $\sim 2$ au from the Sun. While the gas productivity varies greatly during an apparition, the mixing ratios with respect to $\mathrm{H}_{2} \mathrm{O}$ usually remain constant (for a given comet) to a small degree of uncertainty for comets measured near 1 au heliocentric. Comets within 0.8 and beyond $2.5 \mathrm{au}$ heliocentric often show differences from this pattern. For example, our results for early July show that water had an unusually high production rate compared with later dates, perhaps owing to the large release of nearly pure water ice grains.

Table 4 summarizes the molecular production rates for NEOWISE during this observing campaign along with the computed molecular abundance ratios. In Figure 7 we show the temporal variability/stability of the determined molecular abundances in comet NEOWISE. Each panel refers to a different molecule and, as defined in Section 3.2, is associated with a different color. Vertical black dotted lines are introduced to assist visualization of our observing dates. Retrieved molecular mixing ratios in comet NEOWISE are reported with two different symbols: a circle for IRTF observations and a star for Keck observations, and are compared relative to a reference value.

We updated reference values (median and the 25th and 75th percentiles) for mixing ratios in an ensemble of 26 Oort Cloud Comets (OCCs). We adopted the cometary mixing ratios for 10 of the 23 OCCs listed in the taxonomical study of Dello Russo et al. (2016); values for the other 13 comets were replaced with recently revised mixing ratios reported in the taxonomical analysis of Lippi et al., where they reanalyzed data for 20 comets by adopting updated fluorescence models and employing uniform retrieval techniques in a consistent fashion (Lippi et al. 2020, 2021). Together with these 23 Oort Cloud comets, we also included mixing ratios for 3 additional comets present in literature and not included in previous statistical analyses, C/2008 Q3 (Garradd; Lippi et al. 2013), C/2012 K1 PanSTARRS (Roth et al. 2017), and C/2017 E4 Lovejoy (Faggi et al. 2018). In Figure 7 we compare mixing ratios measured in comet NEOWISE with the updated reference values. We show the median values as horizontal full black lines, while shaded gray regions define the area between the 25 th and 75 th percentile of the distribution of measured mixing ratios in these 26 OCCs. Numerical values are also shown.

Molecular mixing ratios measured in comet NEOWISE showed interesting behaviors. We can identify two categories of trace species: (1) molecules whose mixing ratios appeared relatively stable over time and whose variations did not significantly exceed the range of the 25th and 75th percentile interval ( $\mathrm{HCN}, \mathrm{NH}_{3}, \mathrm{C}_{2} \mathrm{H}_{2}, \mathrm{H}_{2} \mathrm{CO}$, and $\mathrm{CO}$ ); and (2) molecules that showed strong variations in their abundance ratios, with highly depleted or enriched values relative to the reference distribution $\left(\mathrm{CH}_{3} \mathrm{OH}, \mathrm{C}_{2} \mathrm{H}_{6}\right.$, and $\left.\mathrm{CH}_{4}\right)$.

Methanol abundances relative to water are shown in the first upper-left plot of Figure 7. During the first two observing runs, $\mathrm{CH}_{3} \mathrm{OH}$ mixing ratios appeared to be severely depleted relative to the reference median value in OCCs, with an average value of $\sim 0.40 \%$, which is well below the 25 th percentile of the distribution of measured $\mathrm{CH}_{3} \mathrm{OH}$ abundances in OCCs. In the following observing runs methanol seemed to reveal abundance ratios that were instead stable and consistent with the reference median value in OCCs. 


\section{Molecular Mixing Ratios relative to water $\bigcirc$ iSHELL/NASA IRTF}
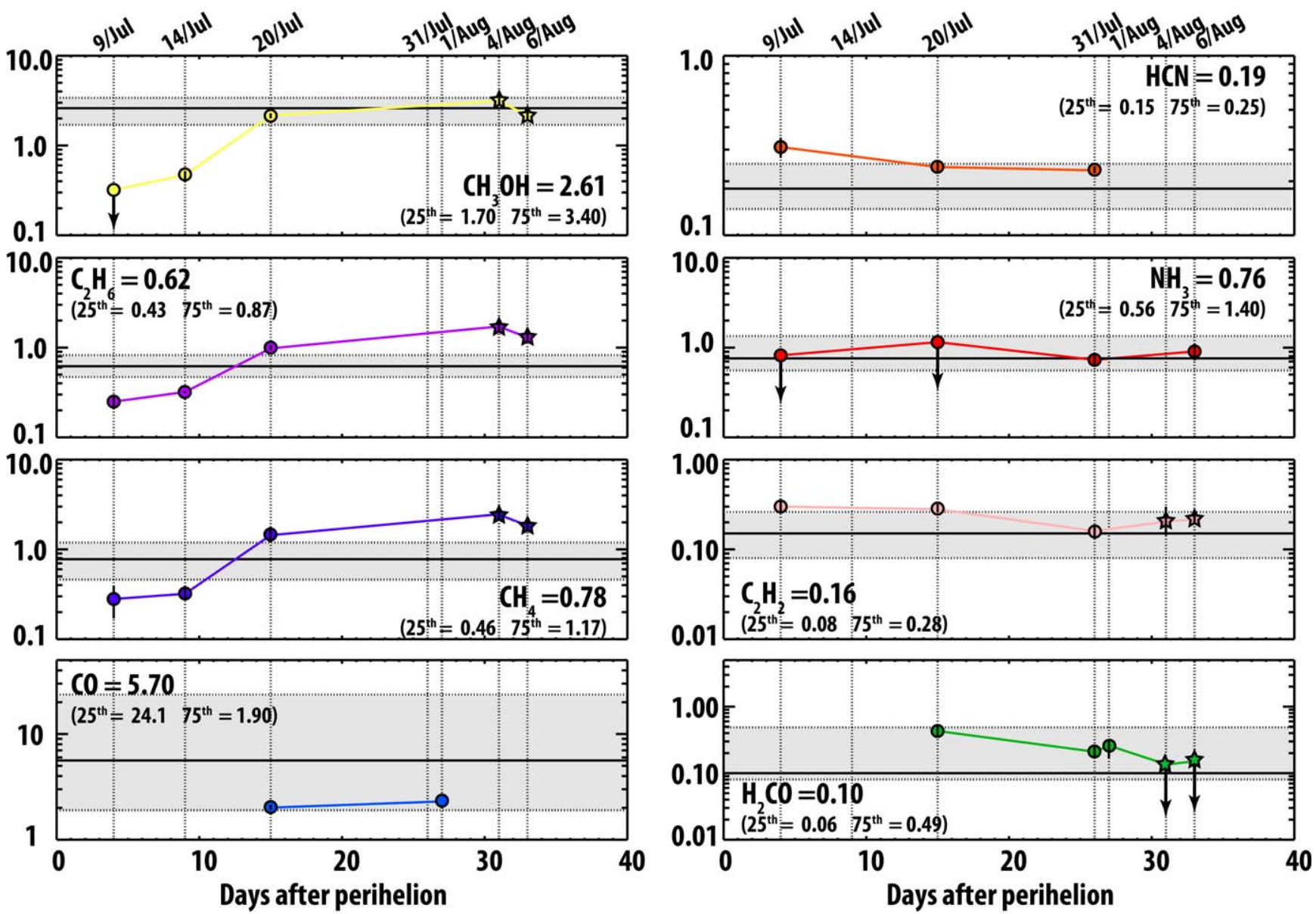

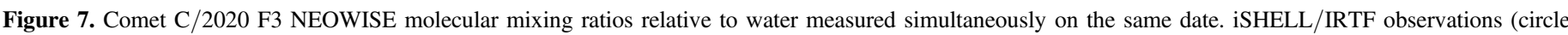

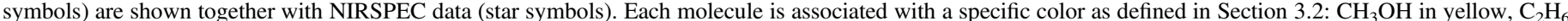

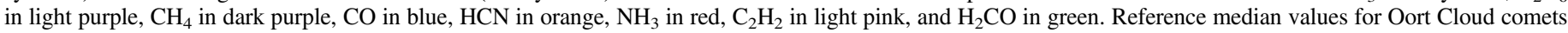

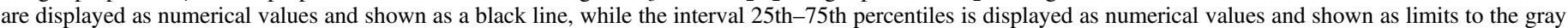
areas.

$\mathrm{C}_{2} \mathrm{H}_{6}$ and $\mathrm{CH}_{4}$ abundance ratios showed behavior similar to methanol-on the first two observing days they both were depleted relative to their reference median values, with average values of $\sim 0.28 \%$ and $\sim 0.30 \%$, respectively, but on the following dates, they showed enriched abundances relative to their reference median values, with average values of $\sim 1.34 \%$ and $\sim 1.85 \%$, respectively.

Five species ( $\mathrm{HCN}, \mathrm{NH}_{3}, \mathrm{C}_{2} \mathrm{H}_{2}, \mathrm{H}_{2} \mathrm{CO}$, and $\mathrm{CO}$ ) instead showed more stable mixing ratios relative to water over the entire observing campaign, with some variation confined within the 25 th-75th percentile interval of the distribution.

$\mathrm{HCN}$ mixing ratios are shown in the first top right panel of Figure 7. The HCN mixing ratio appeared slightly higher during the first observing run, with a value 1.3 times higher than the following dates, but otherwise its abundances remained nearly constant over the span of $\sim 10$ days. Overall $\mathrm{HCN}$ mixing ratios were slightly enriched (with an average value of $0.26 \%$ ) relative to the reference median value of $0.18 \%$, and they appeared to be consistently located at the edge of the 75th percentile of the distribution of measured $\mathrm{HCN}$ mixing ratios in OCCs.

On the first and third observing runs we retrieved $3 \sigma$ upper limits for the $\mathrm{NH}_{3}$ mixing ratios, that were consistent with detections obtained on July 31 and August 6, over about a one-month time interval and were consistent with the reference median value for ammonia in OCCs.

Acetylene abundances measured on the first and third runs appeared slightly higher $(\sim 1.5$ times $)$ relative to later observations. However, despite this small variation, $\mathrm{C}_{2} \mathrm{H}_{2}$ showed nearly stable mixing ratios when compared to other molecules (e.g., $\mathrm{C}_{2} \mathrm{H}_{6}, \mathrm{CH}_{4}$, and $\mathrm{CH}_{3} \mathrm{OH}$ ), with an average mixing ratio of $\sim 0.23 \%$.

Measured $\mathrm{H}_{2} \mathrm{CO}$ abundances on July 20 (the third observing run) appeared 2.7 times higher than on the following dates. However, despite this variation, formaldehyde mixing ratios appeared overall slightly enriched relative to the reference median value of $0.10 \%$.

Finally, CO mixing ratios (with an average value of $\sim 2.16 \%$ relative to water) looked consistently depleted relative to the median reference value, and they are located at the 25 th percentile of measured $\mathrm{CO}$ mixing ratios in OCCs.

\section{Discussion}

\subsection{Evidence for Heterogeneous Composition}

Molecular mixing ratios retrieved by simultaneous measurements of $\mathrm{H}_{2} \mathrm{O}$ and organic trace species provide a reliable metric for investigating the chemical inventory of comets. Even 
though we measured gas in the coma, it might reveal important hints on the composition of nucleus ices.

Cometary nuclei are considered a mixture of refractory and icy material that does not always show the characteristic of being assembled in a uniform homogeneous fashion. Evidence for heterogeneous composition indeed has been observed in cometary comae since $1 \mathrm{P} /$ Halley (e.g., in $\mathrm{H}_{2} \mathrm{CO}$, Mumma \& Reuter 1989), and strong activity variations have been seen in many cometary mixing ratios (e.g., Gibb et al. 2007; A'Hearn et al. 2011; Mumma et al. 2011; Villanueva et al. 2011b; DiSanti et al. 2018; Faggi et al. 2019; Roth et al. 2020). Comets 9P/Tempel-1 and 103P/Hartley 2 (hereafter 103P), as observed by the Deep Impact/EPOXI mission (Feaga et al. 2007; A'Hearn et al. 2011), were the first comets whose nearnucleus gases were mapped in 2D using infrared spectra from space, thereby providing obvious evidence of heterogeneous nuclear composition. The spatial distribution of the 103P coma showed indeed a dichotomy between the water vapor-rich, but $\mathrm{CO}_{2^{-}}$and organics-poor, region arising from the so-called "nucleus waist" and the region at the edge of the smaller lobe of the nucleus that was rich in $\mathrm{CO}_{2}$, organics, and water ice but that had a lower column density of water vapor than the nucleus waist. To mention other examples, from the six observable fragments of comet $\mathrm{C} / 2001$ A2 dramatic changes over the measured mixing ratios of methane and formaldehyde were observed (Gibb et al. 2007). Comet 21P/GiacobiniZinner is also another example of an object with possible heterogeneous chemical composition (Faggi et al. 2019 and references therein).

The rotation of the nucleus might enhance signs of heterogeneous composition, as different regions are activated. However, highly periodic-based observations or luckily the serendipitous recording of dramatic events that produce drastic changes in the comet's activity or in its spatial profiles, such as, for example, strong outbursts or fragmentation, sometimes could reveal precious information about the nucleus composition, revealing hints on its chemical composition. In comet 46P/Wirtanen (hereafter 46P), highly time-cadenced observations of $\mathrm{CN}$ (B-X) emissions recorded at the optical wavelengths showed a periodic variation of radical $\mathrm{CN}$ outgassing over a period of time consistent with the comet nucleus rotation, identifying a possible jet of material in a specific direction (Jehin et al. 2018; CBET 4585). In 46P, recently retrieved ALMA maps showed a periodic outgassing of two methanol jets in a specific direction in the coma (Roth et al. 2020).

Signs of heterogeneous composition in comet C/2020 F3 NEOWISE might be revealed by combining information from its spatial profiles and its mixing ratios. Molecular spatial profiles on July 9, 14, and 20 suggest that icy grains were likely released into the coma, leading to the observed gas excess offset from the dust in the antisunward direction. The molecular abundances show that some molecules $\left(\mathrm{HCN}, \mathrm{NH}_{3}, \mathrm{C}_{2} \mathrm{H}_{2}\right.$, $\mathrm{H}_{2} \mathrm{CO}$, and $\mathrm{CO}$ ), despite small variations, likely followed the water outgassing, revealing mixing ratios relatively constant over the entire observing campaign, while other molecules $\left(\mathrm{CH}_{3} \mathrm{OH}, \mathrm{C}_{2} \mathrm{H}_{6}\right.$, and $\left.\mathrm{CH}_{4}\right)$ showed significant changes in their abundances relative to water. They indeed initially appeared to be depleted relative to reference values for OCCs, on July 9 and 14 , and then they drastically changed into enriched, starting from July 20 (included) and going forward with consecutive observations. This behavior might be an indication of possible nucleus heterogeneous composition.

We could probably think of the nucleus as an object that might have been created by the aggregation of at least two, or likely many, chemically different cometary embryos. Maybe, it might be composed of a first boulder, aggregated from material formed at a certain protoplanetary disk location where $\mathrm{CH}_{3} \mathrm{OH}$, $\mathrm{C}_{2} \mathrm{H}_{6}$, and $\mathrm{CH}_{4}$ ices were enriched, and by a second planetesimal formed at a different location in the disk, which had depletion in such ices instead; or maybe it might be composed of the aggregation of multiple bodies formed at different protoplanetary disk locations, with then different chemical inventories, of which one was severely depleted in $\mathrm{CH}_{3} \mathrm{OH}, \mathrm{C}_{2} \mathrm{H}_{6}$, and $\mathrm{CH}_{4}$ ices. We clearly cannot disentangle such information, and we cannot also extract the evolutional history of this object, but we can possibly try to interpret, in a very simplified view, the strong variation recorded in our measured mixing ratios for $\mathrm{CH}_{3} \mathrm{OH}, \mathrm{C}_{2} \mathrm{H}_{6}$, and $\mathrm{CH}_{4}$ as the likely evidence of outbursts of material sublimating in the coma, from significantly chemically different regions of the nucleus. Or it could be the signature that heated dust grains can release one group of chemicals but not the other.

However, we cannot even exclude that sublimation of nearly pure water-ice grains within $0.56 \mathrm{au}$ of the Sun might have played a significant role. Heterogeneous icy grains may be present throughout all our observations, and we have no information on their distribution and release within the nucleus surface. The heliocentric dependence of various forms of icy grains (apolar ices, polar dirty ice, and polar pure water ice) might explain all the variations we see.

Comparing comet NEOWISE with results obtained in other comets, where asymmetric spatial profiles and drastic changes in their mixing ratios were recorded, we can appreciate the analogies and differences, extracting additional information. In comet C/2007 W1 Boattini and 103P/Hartley 2 (Mumma et al. 2011; Villanueva et al. 2011b), for example, the sublimation of polar and apolar ices, in the form of icy grains, was introduced to explain the asymmetry in the observed spatial profiles. In comet Boattini, spatial distributions of water and methanol indeed, were consistently enhanced along the antisunward direction, and sublimation of polar ices in the coma was hypothesized as the main source of outgassing. Symmetric polar species instead were released from chunks of ice particles that were rotating freely and could emit polar gases with spherical symmetry. In comet $\mathrm{C} / 2013$ V5 (Oukaimeden), dramatic changes in abundance ratios in $\mathrm{C}_{2} \mathrm{H}_{6}$ (about $\sim 87 \%$ ) were recorded, suggesting a nucleus with nonhomogeneous ice composition (DiSanti et al. 2018). $\mathrm{C}_{2} \mathrm{H}_{6}$ spatial profiles showed two distinct behaviors: in one case, they faithfully tracked the water spatial distribution, possibly indicating a polar-dominated phase of ice controlling the outgassing; in the other, they differed substantially from the water spatial distribution. The combination may indicate the presence of both polar- and apolar-dominated ices.

In comet NEOWISE we infer the likely presence of icy grains in the coma, but we do not observe a strict relationship between polar and apolar molecules, perhaps indicating the presence of both icy phases in contributing to the overall gas production. Our interpretation of comet NEOWISE results is based on seven observing runs and, in order to obtain a more exhaustive view of its chemical inventory and evolution, additional observations at higher temporal frequency would 
have been needed both at pre- and postperihelion, but that was not possible.

\subsection{Heritage and Processing History}

The original orbit of the comet NEOWISE showed a semimajor axis of 274.5 au (Nakano note 4202), so it is not a dynamically new comet-it is a long-period comet that has visited the inner solar system on previous apparitions. Its measured composition certainly leaves seeds of thoughts about its formative versus processed composition as revealed by its mixing ratios. What is the meaning of cometary nuclear heterogeneity in terms of cometary formation? And does the measured mixing ratio reflect the conditions of the natal environment or of its later processing history? Answers to these questions are not trivial, and from our analysis, we could attempt to add a new piece to our understanding of the origin and evolution of the prenebular and precometary materials.

If compared with comets having mixing ratios more stable relative to their reference median values, the observed dichotomy in $\mathrm{CH}_{3} \mathrm{OH}, \mathrm{C}_{2} \mathrm{H}_{6}$, and $\mathrm{CH}_{4}$ mixing ratios, which appeared strongly depleted and then became enriched, likely suggests evidence of heterogeneous composition, indicating that perhaps such precometary ices came from two different locations in the protoplanetary disk where they experienced different thermal processing histories.

Our observed enrichment in $\mathrm{CH}_{3} \mathrm{OH}, \mathrm{C}_{2} \mathrm{H}_{6}$, and $\mathrm{CH}_{4}$ mixing ratios reflects the fact that such cometary ices perhaps formed in a cold environment. At low temperatures, indeed $(T \leqslant 30 \mathrm{~K})$ the "freeze-out" mechanism behaves as a selector for accreting hypervolatile species on grain surfaces (e.g., $\mathrm{CO}_{2}, \mathrm{CO}$, and $\mathrm{N}_{2}$ ) and hydrogen addition then becomes a leading process for enhancing molecular complexity (Charnley \& Rodgers 2008). For example, $\mathrm{H}$ addition could explain rich methane abundances via hydrogenation of $\mathrm{C}$ leading to $\mathrm{CH}_{2}$ and finally to $\mathrm{CH}_{4}$, or it could explain rich methanol abundances via hydrogenation of $\mathrm{CO}$ leading to $\mathrm{H}_{2} \mathrm{CO}$ and finally to $\mathrm{CH}_{3} \mathrm{OH}$ (Watanabe et al. 2003a, 2003b). After the formation of $\mathrm{C}_{2} \mathrm{H}_{2}$ in warm photospheric gas reactions of atomic $\mathrm{C}$ with $\mathrm{H}_{2}$, it has been shown that cold $\sim 10 \mathrm{~K}$ surface chemistry of $\mathrm{C}_{2} \mathrm{H}_{2}$ with $\mathrm{H}$ atoms and $\mathrm{OH}$ radicals results in hydrocarbons, such as $\mathrm{C}_{2} \mathrm{H}_{4}$ and $\mathrm{C}_{2} \mathrm{H}_{6}$, but it also leads to the formation of Complex Organic Molecules (COMs; Tielens 1992; Hiraoka et al. 2000a, 2000b; Fuchs et al. 2009; Kobayashi et al. 2017; Chuang et al. 2020). However, if $\mathrm{CH}_{3} \mathrm{OH}$ and $\mathrm{CH}_{4}$ ices are processed by UV radiation, the products can be methyl and methylene radicals $\left(\mathrm{CH}_{3}, \mathrm{CH}_{2}\right)$, which quickly react to form more complex species, such as $\mathrm{C}_{2} \mathrm{H}_{6}$ (Gerakines et al. 2001).

Depletion in $\mathrm{CH}_{3} \mathrm{OH}, \mathrm{C}_{2} \mathrm{H}_{6}$, and $\mathrm{CH}_{4}$ mixing ratios might be a hint of warmer thermal processing. At warm temperatures, hypervolatile species, such as $\mathrm{CO}$, remain in the gas phase leading to their expected depletion as icy components onto grains surfaces (as seen in comet NEOWISE), and because gasphase production of methanol and ethane are energetically inhibited this might be a possible explanation of their depletion in the precometary ices.

Our observed stable depletion of $\mathrm{CO}$ abundances fits both scenarios of depleted/enriched $\mathrm{CH}_{3} \mathrm{OH}, \mathrm{C}_{2} \mathrm{H}_{6}$, and $\mathrm{CH}_{4}$ mixing ratios; it might be either the signature of a warm formative region or a signature of efficient hydrogenation reactions on grain surfaces at cold temperatures that lead to abundant $\mathrm{H}_{2} \mathrm{CO}$ and $\mathrm{CH}_{3} \mathrm{OH}$. However, because comet NEOWISE is not a new dynamical comet, we cannot fully discard that previous apparitions might have shaped the composition of hypervolatiles, so depletion of $\mathrm{CO}$ might also be a possible signature of its thermal history.

\section{Conclusions}

In this paper, we report the detailed analysis and discussion of the molecular composition of comet C/2020 F3 NEOWISE as revealed by high-resolution spectroscopic observations using iSHELL/IRTF and NIRSPEC 2.0/Keck II. We followed the comet from a heliocentric distance of $R_{H}=0.338-0.937$ au and over a geocentric distance ranging from $\Delta=0.963$ to $0.796 \mathrm{au}$ (closest approach to Earth), to 0.918 au.

A plethora of molecules $\left(\mathrm{H}_{2} \mathrm{O}, \mathrm{HCN}, \mathrm{C}_{2} \mathrm{H}_{2}, \mathrm{NH}_{3}, \mathrm{NH}_{2}, \mathrm{C}_{2} \mathrm{H}_{6}\right.$, $\mathrm{CH}_{4}, \mathrm{H}_{2} \mathrm{CO}$, and $\mathrm{CO}$ ) was detected over the entire observing campaign, allowing a comprehensive picture of comet NEOWISE molecular composition and allowing investigations of its evolution over a 1 month time interval. The possible presence of icy grains in the coma was identified through the interpretation of spatial profile analyses. In the first three observing days (July 9, 14, and 20) all the molecular outgassing profiles appeared to be shifted toward the antisunward direction, relative to the dust profiles that are centered on the nucleus-centric position, while in the last four observing runs (July 31 and August 1, 4, and 6) all the gas primary species showed spatial profiles that followed the dust distribution. The observed outgassing excess in the antisunward direction was interpreted as a possible effect of sublimation of icy grains, which dominated the water outgassing from the nucleus. Solar phase angles were close to $90^{\circ}$, allowing the Sun-to-comet vector to be nearly in the plane of the sky and therefore minimizing projection effects on the observed spatial profiles.

Analysis of molecular mixing ratios showed a dichotomy between molecules whose mixing ratios appeared relatively stable over time $\left(\mathrm{HCN}, \mathrm{NH}_{3}, \mathrm{C}_{2} \mathrm{H}_{2}, \mathrm{H}_{2} \mathrm{CO}\right.$, and $\left.\mathrm{CO}\right)$ and those that showed strong variations of their abundance ratios, with highly depleted/enriched values relative to the reference distribution. $\left(\mathrm{CH}_{3} \mathrm{OH}, \mathrm{C}_{2} \mathrm{H}_{6}\right.$, and $\left.\mathrm{CH}_{4}\right)$.

By combining information encoded in comet C/2020 F3 NEOWISE spatial profiles and its mixing ratios, we could interpret the results as signs of heterogeneous composition for its nucleus. The strong variations observed in the mixing ratios for $\mathrm{CH}_{3} \mathrm{OH}, \mathrm{C}_{2} \mathrm{H}_{6}$, and $\mathrm{CH}_{4}$ can be associated with strong changes in outgassing from chemically distinct components of the nucleus. However, we have no information on their distribution within the nucleus surface.

This work was supported by the NASA Astrobiology Institute through award (13-13NAI7-0032) to the Goddard Center for Astrobiology and by the NASA's Planetary Science Division Internal Scientist Funding Program (ISFM) through the Fundamental Laboratory Research (FLaRe) work package. M.J.M. was also supported by NASA Goddard's Emeritus Program. Data presented in this work were obtained at the NASA Infrared Telescope Facility, operated by the University of Hawai'i and at the W. M. Keck Observatory, operated as a scientific partnership among Caltech, UCLA, and NASA. We thank the staff of both IRTF and Keck Observatories for their outstanding operational support and valuable technical suggestions during the observing campaign. The authors also thank the Directors of the NASA InfraRed Telescope Facility and the W. M. Keck Observatory for awarding time to our DDT proposals, allowing investigations of this interesting object. 
The authors wish to recognize and acknowledge the very significant cultural role and reverence that the summit of Maunakea has always had within the indigenous Hawaiian community. We are most fortunate to have the opportunity to conduct observations from this mountain.

\section{ORCID iDs}

Sara Faggi (ib https://orcid.org/0000-0003-0194-5615

Manuela Lippi (i) https://orcid.org/0000-0001-9185-878X

Maria Camarca (1D https://orcid.org/0000-0003-3887-4080

Camillus F. Buzard (iD https://orcid.org/0000-0002-9943-6124

Geronimo L. Villanueva (iD https://orcid.org/0000-0002-

2662-5776

Geoffrey A. Blake (10 https://orcid.org/0000-0003-0787-1610

Michael J. Mumma (D) https://orcid.org/0000-0003-4627-750X

\section{References}

A'Hearn, M. F., Belton, M. J. S., Delamere, W. A., et al. 2011, Sci, 332, 1396 Altwegg, K., Balsiger, H., Bar-Nun, A., et al. 2016, SciA, 2, 1600285

Altwegg, K., Balsiger, H., Hänni, N., et al. 2020, NatAs, 4, 533

Beer, E. H., Beer, E. H., Podolak, M., et al. 2006, Icar, 180, 473

Bergin, E. A., Aikawa, Y., Blake, G. A., \& van Dishoeck, E. F. 2007 Protostars and Planets V (Tucson, AZ: Univ. Arizona Press.), 751

Bergin, E. A., \& Cleeves, L. I. 2018, Handbook of Exoplanets, Vol. 786 (Cham: Springer), 137

Bergner, J. B., Oberg, K. I., Rajappan, M., \& Fayolle, E. C. 2016, ApJ, 829, 85, (IOP Publishing)

Bertaux, J.-L., Costa, J., Mäkinen, T., et al. 1999, P\&SS, 47, 725

Bockelée-Morvan, D., Crovisier, J., Mumma, M. J., \& Weaver, H. A. 2004, Comets II (Tucson, AZ: Univ. Arizona Press), 391

Bonev, B. P. 2005, PhDT, The Univ. of Toledo 322

Bonev, B. P., Mumma, M. J., Dello Russo, N., et al. 2004, ApJ, 615, 1048

Bonev, B. P., Mumma, M. J., Gibb, E. L., et al. 2009, ApJ, 699, 1563

Charnley, S. B., \& Rodgers, S. D. 2008, SSRv, 138, 59

Chuang, K. J., Fedoseev, G., Qasim, D., et al. 2020, A\&A, 635, A199

Cleeves, L. I., Bergin, E. A., Alexander, C. M. O., et al. 2014, Sci, 345, 1590

Combi, M. R., Mäkinen, T., Bertaux, J., Quemerais, E., \& Ferron, S. 2021, ApJL, 907, L38

Dello Russo, N., DiSanti, M. A., Mumma, M. J., Magee-Sauer, K., \& Rettig, T. W. 1998, Icar, 135, 377

Dello Russo, N., Kawakita, H., Vervack, R. J., \& Weaver, H. A. 2016, Icar, 278, 301

DiSanti, M. A., Bonev, B. P., Gibb, E. L., et al. 2018, AJ, 156, 258

DiSanti, M. A., Bonev, B. P., Magee-Sauer, K., et al. 2006, ApJ, 650, 470

DiSanti, M. A., Mumma, M. J., Dello Russo, N., \& Magee-Sauer, K. 2001, Icar, 153, 361

Eistrup, C., Walsh, C., \& van Dishoeck, E. F. 2019, A\&A, 629, A84

Faggi, S., Mumma, M. J., Villanueva, G. L., Paganini, L., \& Lippi, M. 2019, AJ, 158,254

Faggi, S., Villanueva, G. L., Mumma, M. J., \& Paganini, L. 2018, AJ, 156, 68

Feaga, L. M., A'Hearn, M. F., Sunshine, J. M., Groussin, O., \& Farnham, T. L. 2007, Icar, 190, 345

Fuchs, G. W., Cuppen, H. M., Ioppolo, S., et al. 2009, A\&A, 505, 629

Garrod, R. T. 2019, ApJ, 884, 69
Gerakines, P. A., Moore, M. H., \& Hudson, R. L. 2001, JGRE, 106, 33381 Gerakines, P. A., Moore, M. H., \& Hudson, R. L. 2004, Icar, 170, 202

Gelaro, Ronald, McCarty, Will, Suárez, Max J., et al. 2017, JCli, 30, 5419

Gibb, E. L., DiSanti, M. A., Magee-Sauer, K., et al. 2007, Icar, 188, 224

Hiraoka, K., Sato, T., \& Takayama, T. 2000b, in IAU Symp. 197, Astrochemistry: From Molecular Clouds to Planetary Systems (Cambridge: Cambridge Univ. Press), 283

Hiraoka, K., Takayama, T., Euchi, A., Handa, H., \& Sato, T. 2000a, ApJ, 532,1029

Irvine, W. M., Schloerb, F. P., Crovisier, J., Fegley, B. J., \& Mumma, M. J. 2000, in Protostars and Planets IV, ed. V. Mannings (Tucson, AZ: Univ. Arizona Press), 1159

Jehin, E., Moulane, Y., Manfroid, J., \& Pozuelos, F. 2018, Central Bureau for Astronomical Telegrams, CBET, 4585

Jewitt, D., Morbidelli, A., \& Rauer, H. 2007, Trans-Neptunian Objects and Comets (Berlin: Springer), 258

Kawakita, H., \& Mumma, M. J. 2011, ApJ, 727, 91

Kobayashi, H., Hidaka, H., Lamberts, T., et al. 2017, ApJ, 837, 155

Lippi, M., Villanueva, G. L., DiSanti, M. A., et al. 2013, A\&A, 551, A51

Lippi, M., Villanueva, G. L., Mumma, M. J., et al. 2020, AJ, 159, 157

Lippi, M., Villanueva, G. L., Mumma, M. J., \& Faggi, S. 2021, AJ, 162, 74

Mäkinen, J., \& Combi, M. R. 2005, Icar, 177, 217

Martin, E. C., Fitzgerald, M. P., McLean, I. S., et al. 2018, Proc. SPIE, 10702, 107020A

McLean, I. S., Becklin, E. E., Bendikse, O., et al. 1998, Proc. SPIE, 3354, 566

Morbidelli, A., \& Rickman, H. 2015, A\&A, 583, A43

Mumma, M., Charnley, S., Cordiner, M., et al. 2019, EPSC-DPS Joint Meeting 2019, 13, 1916

Mumma, M. J., et al. 2011, ApJL, 734, L7

Mumma, M. J., Bonev, B. P., Villanueva, G. L., et al. 2011, ApJL, 734, L7

Mumma, M. J., Charnley, S., Cordiner, M., et al. 2018, AAS, 50, 209.02

Mumma, M. J., \& Charnley, S. B. 2011, ARA\&A, 49, 471

Mumma, M. J., \& Reuter, D. C. 1989, ApJ, 344, 940

Mumma, M. J., Weissman, P. R., \& Stern, S. A. 1993, Protostars and Planets III (Tucson, AZ: Univ. Arizona Press), 1177

Poch, O., Istiqomah, I., Quirico, E., et al. 2020, Sci, 367, 6483

Rayner, J., Bond, T., Bonnet, M., et al. 2012, Proc. SPIE, 8446, 84462C

Roth, N., Milam, S., Cordiner, M., et al. 2021, PSJ, 2, 55

Roth, N. X., Gibb, E. L., Bonev, B. P., et al. 2017, AJ, 153, 168

Shinnaka, Y., \& Kawakita, H. 2016, AJ, 152, 145

Tielens, A. G. G. M. 1992, Chemistry and Spectroscopy of Interstellar Molecules Edited by KK Bohme (Tokyo: Univ. of Tokyo Press), 237

Tielens, A. G. G. M. 2013, RvMP, 85, 1021

Villanueva, G. L., DiSanti, M. A., Mumma, M. J., Xu, L.-H., \& Xu, L.-H. 2012b, ApJ, 747, 1

Villanueva, G. L., Mumma, M. J., Bonev, B. P., et al. 2012a, JQSRT, 113, 202

Villanueva, G. L., Mumma, M. J., DiSanti, M. A., et al. 2011b, Icar, 216, 227

Villanueva, G. L., Mumma, M. J., DiSanti, M. A., et al. 2012c, Icar, 220, 291

Villanueva, G. L., Mumma, M. J., \& Magee-Sauer, K. 2011a, JGRE, 116, E08012

Villanueva, G. L., Mumma, M. J., Novak, R. E., et al. 2013, Icar, 223, 11

Villanueva, G. L., Smith, M. D., Protopapa, S., Faggi, S., \& Mandell, A. M. 2018, arXiv: 1803.02008

Wang, H., Bell, R. C., Iedema, M. J., Tsekouras, A. A., \& Cowin, J. P. 2005, ApJ, 620, 1027, (IOP Publishing)

Watanabe, N., Shiraki, T., \& Kouchi, A. 2003a, ApJ, 588, L12

Watanabe, N., Shiraki, T., \& Kouchi, A. 2003b, in IAU Meeting 25, Formation of Cometary Material (Cambridge: Cambridge Univ. Press), E34

Xie, X., \& Mumma, M. J. 1996, ApJ, 464, 457 